\title{
Identification and Structural Characterization of Unidentified Impurity in Bisoprolol Film-Coated Tablets
}

\author{
Ivana Mitrevska, Ema Kikovska-Stojanovska, Gjorgji Petrusevski, Marina Chachorovska, \\ Suzan Memed-Sejfulah, and Sonja Ugarkovic
}

Research \& Development, Alkaloid AD Skopje, Blvd. Aleksandar Makedonski 12, 1000 Skopje, Macedonia

Correspondence should be addressed to Ivana Mitrevska; imickoska@alkaloid.com.mk

Received 26 November 2016; Revised 12 January 2017; Accepted 31 January 2017; Published 23 March 2017

Academic Editor: José Bernal

Copyright (c) 2017 Ivana Mitrevska et al. This is an open access article distributed under the Creative Commons Attribution License, which permits unrestricted use, distribution, and reproduction in any medium, provided the original work is properly cited.

\begin{abstract}
The aim of this study is the identification, structural characterization, and qualification of a degradation impurity of bisoprolol labeled as Impurity RRT 0.95 . This degradation product is considered as a principal thermal degradation impurity identified in bisoprolol film-coated tablets. The impurity has been observed in the stress thermal degradation study of the drug product. Using HPLC/DAD/ESI-MS method, a tentative structure was assigned and afterwards confirmed by detailed structural characterization using NMR spectroscopy. The structure of the target Impurity RRT 0.95 was elucidated as phosphomonoester of bisoprolol, having relative molecular mass of 406 (positive ionization mode). The structural characterization was followed by qualification of Impurity RRT 0.95 using several different in silico methodologies. From the results obtained, it can be concluded that no new structural alerts have been generated for Impurity RRT 0.95 relative to the parent compound bisoprolol. The current study presents an in-depth analysis of the full characterization and qualification of an unidentified impurity in a drug product with the purpose of properly defining the quality specification of the product.
\end{abstract}

\section{Introduction}

The identification, structure elucidation, and quantitative determination of impurities and degradation products are of prime importance in the course of all phases of research, development, and production of drug formulations [1]. In order to establish the stability indicating nature of a formulation, knowledge should be gained on the impurity profile of the bulk drug material used for the development of the formulation, in particular the synthesis-related impurities versus degradation products [2]. Instrumental in that sense are preliminary stability studies carried out with the bulk drug material as well as forced degradation/stress studies.

Identification, qualification, and quantification of impurities are critical tools for assessing the safety and quality of pharmaceutical drug substances and their associated dosage forms.

Bisoprolol is a highly beta ${ }_{1}$-selective-adrenoceptor blocking agent used for the treatment of hypertension, angina pectoris, and heart failure. Treatment with bisoprolol is generally a long-term treatment that requires continuous daily intake of the medicine. Therefore, the quality of the product maintained through its entire shelf life is of utmost importance on the path of delivering efficient and safe therapy to the patients.

In the present study, throughout the long-term $\left(25^{\circ} \mathrm{C} /\right.$ $60 \% \mathrm{RH})$ and accelerated $\left(40^{\circ} \mathrm{C} / 75 \% \mathrm{RH}\right)$ stability course of generic bisoprolol film-coated tablets, an increase of a particular degradation product analyzed with HPLC at RRT 0.95 was observed. The degradation product is evident for all strengths of the drug product, though with the most prominent increase for the lowest bisoprolol $2.5 \mathrm{mg}$ strength. Testing at accelerated conditions as the most stressful scenario for the product generated degradation levels up to $0.47 \%$ (by area percentage) for the respective Impurity RRT 0.95 .

It should be highlighted that, according to ICH Q3B(R2), there is an identification threshold for a structurally 
unknown/unidentified impurity (related to the total daily intake), for the respective product being $0.2 \%$. When the levels of the respective impurity (RRT 0.95 in this case) are exceeded throughout the stability study, the impurity should be structurally identified.

Upon detailed analysis, it was concluded that the degradation product derived from the interaction of bisoprolol and the excipient calcium hydrogen phosphate. In order to perform structural identification and subsequently qualification of the given degradation product, a sufficient quantity was isolated and using mass spectrometry and NMR spectroscopy structurally characterized to be phosphomonoester of bisoprolol.

In silico approach was undertaken to qualify this impurity in relation to the genotoxicological profile of the parent compound, bisoprolol [3]. The analysis performed revealed no new structural alerts of Impurity RRT 0.95 relative to the parent compound bisoprolol.

There are many implications from the presented study where full characterization of the molecular structure and toxicological preferences of an unknown impurity are presented, primarily from an aspect of defining the quality and safety aspects for the drug product of interest. From the listed excipients used in the various bisoprolol oral drug products with marketing authorizations in $\mathrm{ICH}$ countries, it is evident that there are a vast number of products that include calcium hydrogen phosphate as an excipient in the formulation [4]. Therefore, this study might be of particular interest for extended evaluations of the stability and thus the safety profile of different bisoprolol products and leaves further possibilities for shelf-life extensions of the product with plausible commercial aspects in that manner.

\section{Experimental Details}

2.1. Materials. Bisoprolol fumarate working standard and bisoprolol $2.5 \mathrm{mg}$ film-coated tablets, orthophosphoric acid $85 \%$, hydrogen peroxide $30 \%$, acetonitrile, and formic acid were obtained from Merck GmbH, Germany. All chemicals including water were of analytical grade and were used without further purification.

\subsection{Instrumentation}

2.2.1. HPLC Analysis. The HPLC method for related substances was adopted from the Ph.Eur monograph for bisoprolol fumarate drug substance (Supplement 6.1 04/2008: 1710) [5]. High performance liquid chromatography was performed on a Waters Alliance PDA HPLC system. The column Nucleosil 100-5 C18 HD, $250 \mathrm{~mm} \times 4.6 \mathrm{~mm}, 5 \mathrm{~m}$, E.C., was maintained at $30^{\circ} \mathrm{C}$, with UV detector wavelength of $225 \mathrm{~nm}$. The gradient involves two mobile phases consisting of $10 \mathrm{~g} /$ $\mathrm{L}$ solution of orthophosphoric acid in water as solvent A and $10 \mathrm{~g} / \mathrm{L}$ solution of orthophosphoric acid in acetonitrile as solvent $\mathrm{B}$. The flow rate was set to $1.0 \mathrm{~mL} / \mathrm{min}$. The gradient program was employed with a timed gradient program of $\mathrm{T}(\mathrm{min}) / \% \mathrm{~B}(\mathrm{v} / \mathrm{v}): 0.01 / 0,0 / 10,35 / 80,40 / 10,50 / 10$, for the separation. The analysis run time was $50 \mathrm{~min}$; the injection volume was $10 \mu \mathrm{L}$. The obtained results were evaluated by the Empower 3 software. Typical chromatograms of bisoprolol fumarate working standard and film-coated tablets are shown in Figure 1.

2.2.2. HPLC/MS Analysis. HPLC/MS analysis was performed on the 1100 Infinity series diode array and mass detector in series (Agilent Technologies, Germany). The mass detector was an Agilent G2449A Ion-Trap Mass Spectrometer equipped with an electrospray ionization (ESI) system and controlled by LCMSD software (Agilent, v.6.2). Nitrogen was used as nebulizing gas at pressure of 50 psi and flow was adjusted to $12 \mathrm{~L} \mathrm{~min}^{-1}$. The heated capillary and the voltage were maintained at $325^{\circ} \mathrm{C}$ and $3500 \mathrm{~V}$, respectively. MS data were acquired in the positive ionization mode. The full scan covered the mass range at $m / z 70-800$. Collision-induced fragmentation experiments were performed in the ion trap using helium as collision gas, with voltage ramping cycle from 0.3 up to $2 \mathrm{~V}$. Maximum accumulation time of ion trap and the number of MS repetitions to obtain the MS average spectra were set at $300 \mathrm{~ms}$ and 3, respectively. It is well known that phosphoric acid is a nonvolatile reagent and is not compatible with LC/MS analysis. For that reason, a new reagent (formic acid) suitable for LC/MS analysis was included and the method was developed [6-10].

HPLC/MS method was slightly modified in order to be suitable for the MS detector by changing the mobile phase to water formic acid-acetonitrile. The gradient involves two mobile phases consisting of water and formic acid as solvent $\mathrm{A}$ in the ratio $99 \%: 1 \%(\mathrm{v} / \mathrm{v})$ and acetonitrile and formic acid as solvent $\mathrm{B}$ in the ratio $99 \%: 1 \%(\mathrm{v} / \mathrm{v})$. The gradient program was employed with a timed gradient program of $\mathrm{T}(\mathrm{min}) / \% \mathrm{~B}(\mathrm{v} / \mathrm{v}): 0.01 / 0,0 / 10,35 / 80,40 / 10,50 / 10$, for the separation. Flow rate was kept at $0.8 \mathrm{~mL} / \mathrm{min}$, with UV detector wavelength of $270 \mathrm{~nm}$ and injection volume of $50 \mu \mathrm{L}$. The scanning wavelength was set at $270 \mathrm{~nm}$ (in the original method being $225 \mathrm{~nm}$ ) because of the observed spectral interferences caused by the formic acid at lower wavelengths. The retention times for the monitored samples obtained are given in the chromatograms and they are $16.2 \mathrm{~min}$ for bisoprolol and $15.9 \mathrm{~min}$ for the targeted impurity (Figure 2). These changes have caused a slight increase in relative retention time (RRT) of unknown impurity on 0.98 , which was confirmed by comparison of the chromatograms (Figures 1 and 2) and UV spectra (Figure 3) obtained by the two methods. The spectra were extracted from a PDA detector in the range of $200-400 \mathrm{~nm}$.

2.2.3. NMR Spectroscopy. Nuclear magnetic resonance (NMR) measurement was performed on Avance I (Bruker) spectrometer operating at $400.13 \mathrm{MHz}\left({ }^{1} \mathrm{H}\right)$, equipped with a $5 \mathrm{~mm}$ QNP probe head, or an Avance II (Bruker) spectrometer operating at $700 \mathrm{MHz}\left({ }^{1} \mathrm{H}\right)$, equipped with a cryoprobe and a field gradient delivering z-field gradients. All experiments were performed at room temperature. Topspin software (Bruker) was used for acquisition and NMR notebook for data processing and plotting. 


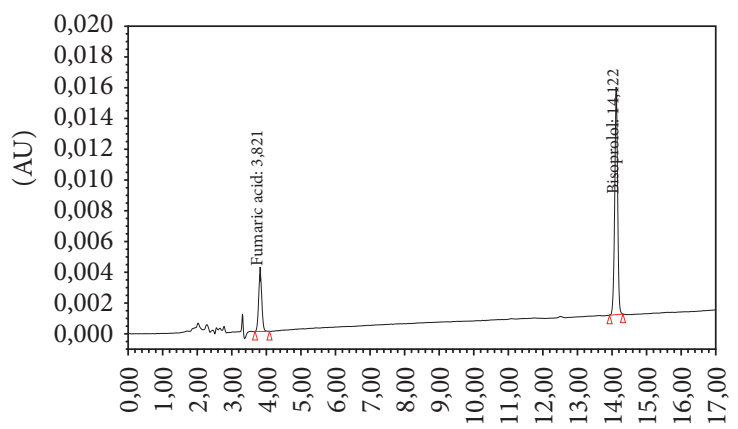

(Minutes)

(a) HPLC chromatogram of bisoprolol fumarate working standard

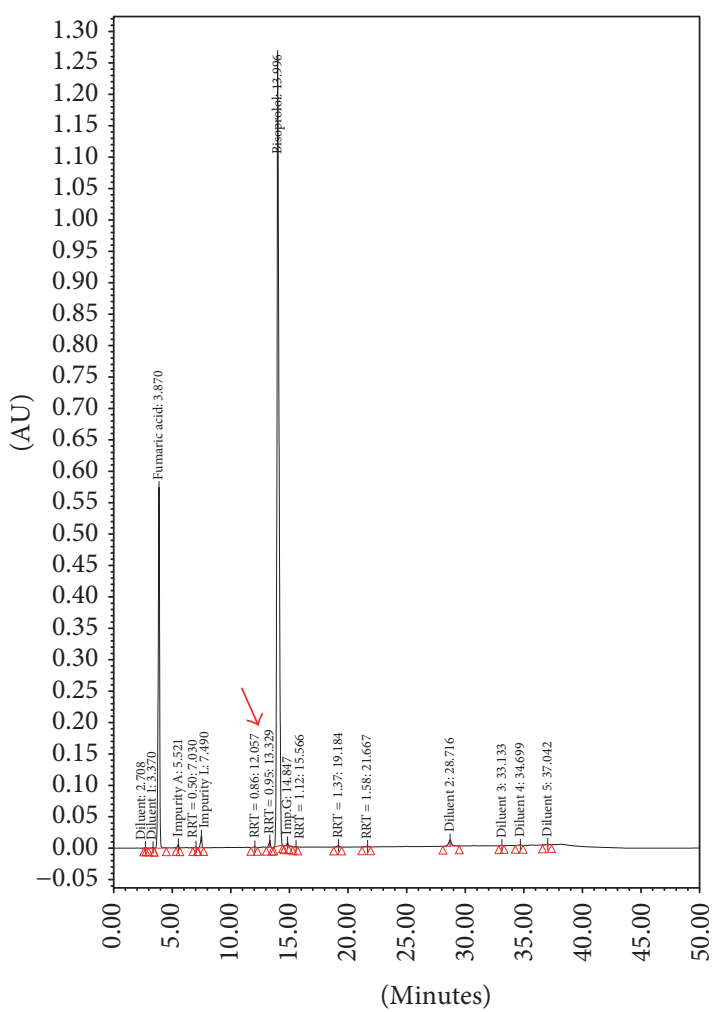

(b) HPLC chromatogram of drug product during the shelf-life stability program

FIgURE 1: Typical HPLC chromatograms of (a) bisoprolol fumarate working standard and (b) film-coated tablets according to Ph.Eur monograph for bisoprolol fumarate drug substance (Supplement 6.1 04/2008: 1710).

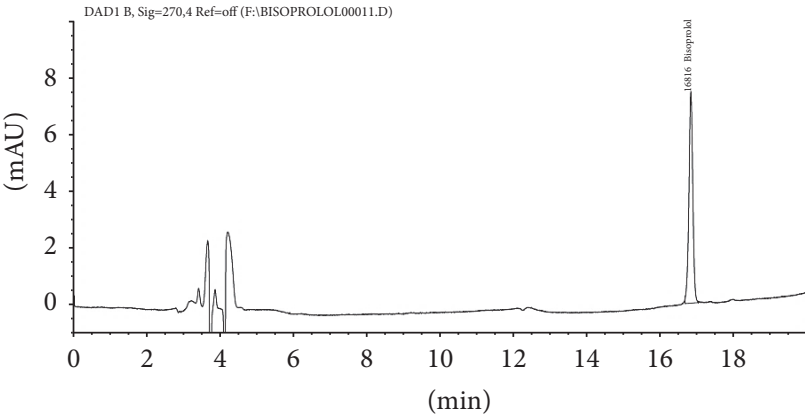

(a) HPLC/MS chromatogram of bisoprolol fumarate working standard

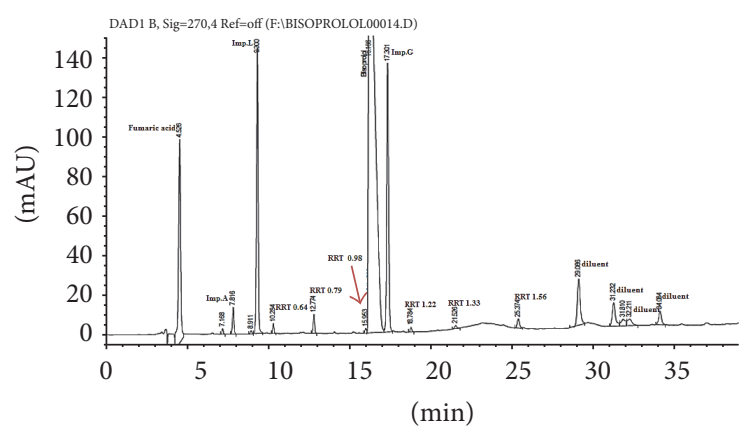

(b) HPLC/MS chromatogram of bisoprolol $2.5 \mathrm{mg}$ film-coated tablets

FIgURE 2: Typical HPLC/MS chromatograms of (a) bisoprolol fumarate working standard and (b) film-coated tablets according to HPLC/DAD/ESI-MS method.

2.3. Preparation of Standard and Test Solutions. A standard solution containing $0.005 \mathrm{mg} / \mathrm{mL}$ bisoprolol fumarate was prepared in a mixture of water and acetonitrile at the ratio $80 \%: 20 \%(\mathrm{v} / \mathrm{v})$ and test solution containing $1 \mathrm{mg} / \mathrm{mL}$ bisoprolol fumarate.

2.4. Stability Studies. During the stability study, the product is maintained at defined conditions of temperature and humidity and analyzed periodically to establish whether changes in the physical and chemical characteristics occur. Stability studies are conducted in compliance with ICH Q1A (R2) guideline [11].

\subsubsection{Long-Term Stability Studies}

Storage Conditions. Storage conditions were as follows: temperature, $25^{\circ} \mathrm{C}\left( \pm 2^{\circ} \mathrm{C}\right)$; relative humidity, $60 \% \mathrm{RH}( \pm 5 \%)$; 


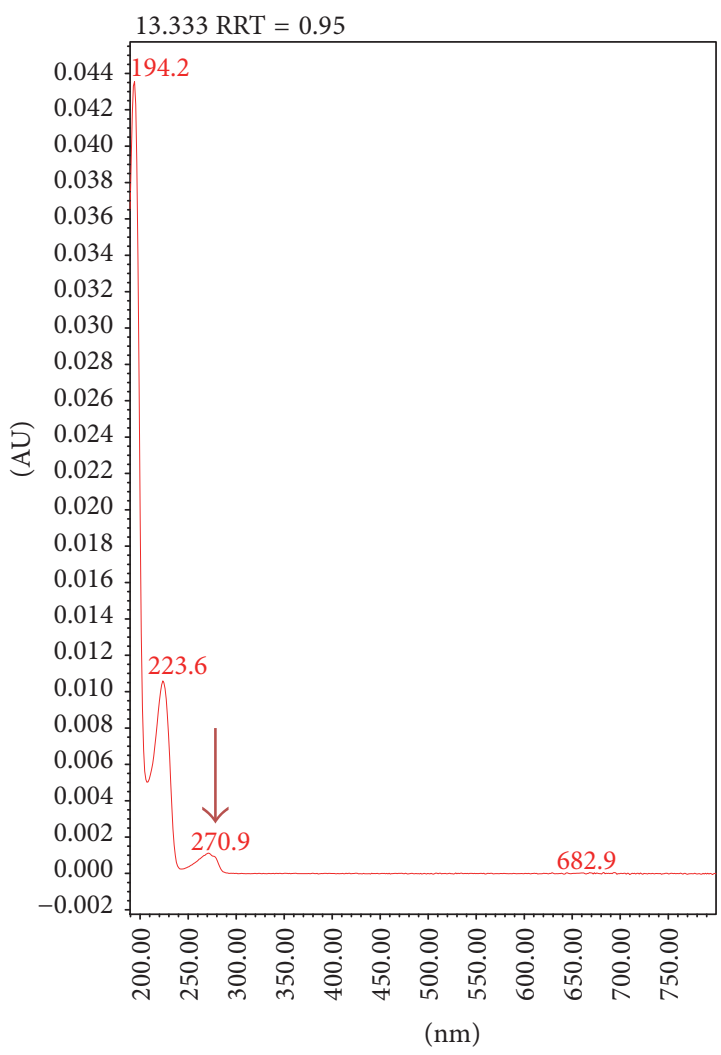

(a)

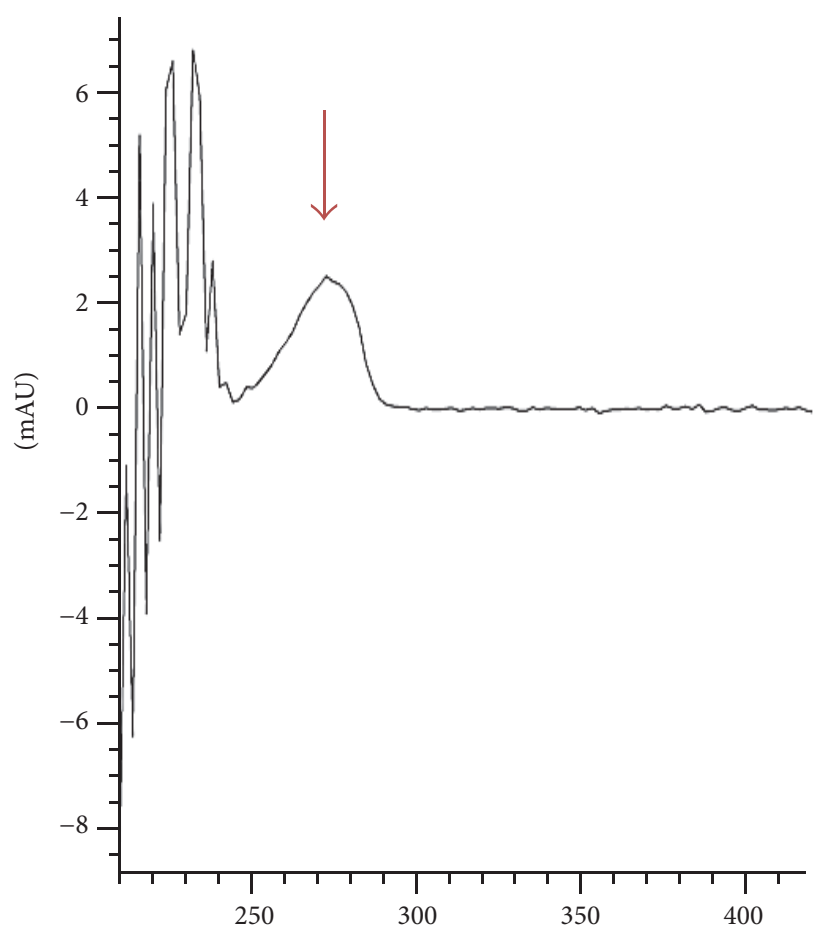

(b)

FIgURE 3: (a) UV spectra of Imp. RRT 0.95 obtained by original HPLC method; (b) UV spectra of Imp. RRT 0.95 obtained by HPLC/MS method.

storage period, minimum 24 months; frequency, every 3 months during the first year and every 6 months during the second year.

\subsubsection{Accelerated Studies}

Storage Conditions. Storage conditions were as follows: temperature, $40^{\circ} \mathrm{C}\left( \pm 2^{\circ} \mathrm{C}\right)$; relative humidity, $75 \% \mathrm{RH}( \pm 5 \%)$; storage period, 6 months; frequency, every 3 months.

2.5. Force Degradation Studies. In order to check the possibility of accelerating the rate of degradation of the amount of this impurity, forced degradation studies were performed. The stress conditions were set as follows.

2.5.1. Photostability Degradation Study. Film-coated tablets were exposed to light providing an illumination of 1.2 million lux-hours $(7.1 \mathrm{~h})$ and an integrated near-ultraviolet energy of $200 \mathrm{Wh} / \mathrm{m}^{2}(2.9 \mathrm{~h})$ and then exposed to five times increased irradiance dose $(35 \mathrm{~h})$.

2.5.2. Oxidative Degradation Study. Film-coated tablets were treated with hydrogen peroxide solution $(2 \%$ in water, $5 \mathrm{~mL})$ at $50^{\circ} \mathrm{C}$ (water bath) for 3 hours, and then samples were cooled and analyzed as per the proposed method.

2.5.3. Thermal/Humidity Degradation Study. Binary mixtures of bisoprolol fumarate with various excipients were placed on a quartz Petri dish and directly exposed to $40^{\circ} \mathrm{C} / 75 \% \mathrm{RH}$, for 2 , 4 , and 8 weeks. Samples were analyzed as per the proposed method.

Thermal Degradation Study A. A film-coated tablets binary mixture as a formulation mass ratio of bisoprolol fumarate and calcium hydrogen phosphate anhydrous was placed on an open quartz Petri dish and kept at $80^{\circ} \mathrm{C}$ for 5 hours, for 5 days. After that, the samples were cooled and analyzed as per the proposed method.

Thermal Degradation Study B. A binary mixture as equal mass ratio of bisoprolol fumarate and calcium hydrogen phosphate anhydrous was placed in an open quartz Petri dish and kept at $80^{\circ} \mathrm{C}$ for 72 hours.

\subsection{Toxicological Aspects}

2.6.1. Toxtree v.2.6.13. Toxtree is a Java-based open-source application that has been developed by IDEA Consult Ltd. 
under the terms of a contract with the Institute for Health and Consumer Protection as part of the JRC at the European Commission.

Toxtree is able to estimate toxic hazards by applying a decision tree approach [12-14]. It includes several decision tree methods out of which relevant to mutagenicity/carcinogenicity are the following:

(i) A decision tree for estimating carcinogenicity (genotoxic and nongenotoxic) and mutagenicity, by discriminant analysis and structural rules [12]

(ii) A decision tree for estimating in vitro mutagenicity (Ames test) [13]

(iii) Structure alerts for the in vivo micronucleus assay in rodents presenting a coarse-grain filter for preliminary screening of potentially in vivo mutagens [14]

2.6.2. TEST Software. Toxicity Estimation Software Tool (TEST) has been developed by the US Environmental Protection Agency as a statistical based tool using several QSAR methodologies to estimate toxicity from molecular structure [15]. TEST v.4.1 used in the present study is an open-source Java library for computational chemistry. It includes models for several endpoints, out of which Ames mutagenicity was used in the current study. TEST allows for estimating toxicity values using several different advanced Quantitative Structure Activity Relationship (QSAR) methodologies [16]: hierarchical method, FDA method, group contribution method, nearest neighbor method, consensus method, and the random forest method [17]. TEST provides multiple validated prediction methodologies so that one can have greater confidence in the predicted toxicities (assuming the predicted toxicities are fairly similar from different methods). In the statistical external validation of the Ames mutagenicity endpoint, the consensus method achieved the best prediction accuracy (concordance) and prediction coverage.

2.6.3. QSAR Toolbox Software. QSAR Toolbox is an in silico tool developed by LMC ASIS in collaboration with OECD and ECHA. This software application is intended for assessing the hazards of chemicals by the following key features: identification of relevant structural characteristics and potential mechanism or mode of action of a target chemical, identification of other chemicals that have the same structural characteristics and/or mechanism or mode of action (categorizing of chemicals), and use of existing experimental data to fill the data gap(s). In order to assess the toxicological hazard, in the present study, the parent compound and the relevant impurity have been profiled for relevant toxicity-related endpoints. Profilers relevant to genetic toxicity and carcinogenicity deriving from structural alerts associated with toxicity include carcinogenicity (genotoxic and nongenotoxic) by ISS, DNA alerts for Ames by OASIS, DNA alerts for CA and MNT by OASIS, in vitro mutagenicity (Ames test) by ISS, in vivo chromosomal mutation, and OncoLogic primary classification. Upon the initial profiling of the relevant structures, data gathering was performed from available databases relevant to genetic toxicology and carcinogenicity. When relevant toxicological data is not available in the databases used, the Toolbox can be used for the data gap filling process via definition of category and subcategory of structural analogues and further read-across analysis for determining the final toxicological hazard.

\section{Results and Discussion}

The first step of impurity profiling is the detection of the impurity. Stability studies made on film-coated tablets have shown that the amount of unidentified impurity with RRT 0.95 is increased above the regulatory recommended levels for longer shelf life of the product. It should be highlighted that it is a mandatory requirement from regulatory authorities to identify and characterize any unknown impurity present at a level as low as $0.2 \%$. In order to have complete molecular structure characterization of the studied impurity by NMR spectroscopy, the impurity must be separated from the parent compound by comprehensive separation methods such as preparative liquid chromatography.

In order to check the possibility of accelerating the rate of increase of the amount of this impurity in the studied formulation, trial forced degradation studies were performed. Therefore, the next step was the design of an appropriate force degradation study (stress testing) on the basis of degradation pathways of drug products; discernment of degradation products in formulations that are related to drug substances versus those that are related to nondrug substances (e.g., excipients); structure elucidation of degradation products; determination of the intrinsic stability of a drug substance molecule in solution and solid state; and revelation of the thermolytic degradation mechanism of the drug product [18, 19].

Additionally, a dedicated study was conducted in order to isolate and concentrate the entitled impurity. For the purpose of the experiment, a set of binary mixtures with each excipient was prepared. All binary mixtures were exposed at $40^{\circ} \mathrm{C} / 75 \%$ RH chamber and analyzed by HPLC after 2,4 , and 8 weeks. After 8 weeks, the obtained results showed that the only increase of the amount of RRT 0.95 was found in the binary mixture of bisoprolol fumarate and calcium hydrogen phosphate, up to $0.08 \%$ (Table 1 ).

The obtained results for the unidentified impurity with RRT 0.95 were low; hence, further stress conditions were set.

The next step in this part of the experiment was to set the binary mixture of bisoprolol fumarate and calcium hydrogen phosphate exposed at a temperature of $80^{\circ} \mathrm{C}$ for 121 hours in an open Petri dish.

The result for unidentified impurities was $1.07 \%$, with also a satisfactory amount of degradation that would allow continuing the experiment and the stress study.

3.1. Force Degradation Study on Film-Coated Tablets. To accelerate the rate of increase in the percentage of unidentified impurities with RRT 0.95, a forced degradation study of the finished pharmaceutical product was performed. The stress conditions were performed as described in Section 2.5 (photostability, oxidative, and thermal degradation A). 
TABLE 1: Results for unidentified impurity with RRT 0.95 in binary mixtures, exposed at $40^{\circ} \mathrm{C} / 75 \% \mathrm{RH}$.

\begin{tabular}{|c|c|c|c|c|}
\hline Bisoprolol fumarate + & Initial & 2 weeks & 4 weeks & 8 weeks \\
\hline Diluent 1 & 1 & 1 & 1 & 1 \\
\hline Diluent 2 (calcium hydrogen phosphate) & l & $0.04 \%$ & $0.05 \%$ & $0.08 \%$ \\
\hline Glidant & I & l & l & l \\
\hline Disintegrant & l & I & I & l \\
\hline Filler & I & l & l & l \\
\hline
\end{tabular}

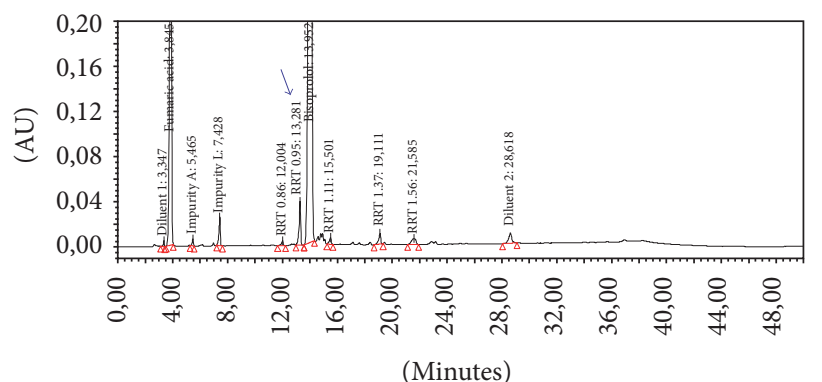

FIgURE 4: HPLC chromatograms of film-coated tablets in thermal degradation studies at $80^{\circ} \mathrm{C}$ during a period of 5 days.

During the photostability degradation of the film-coated tablets, Impurity RRT 0.95 increased up to $0.09 \%$.

In the forced oxidative degradation study, $0.29 \%$ of the unidentified impurity with RRT 0.95 was found in the drug products.

Regarding the thermal degradation, the HPLC trace of the studied impurity with RRT 0.95 in the film-coated tablets showed an increase up to $0.50 \%$.

The results obtained from the initial forced degradation experiments (discussed above) have shown that the preferred method to increase the concentration of the impurity with RRT 0.95 would be to perform prolonged thermal degradation studies of drug product at $80^{\circ} \mathrm{C}$. The time of exposure was set at 5 days (Figure 4 ) and the amount of RRT 0.95 before and after the treatment is disclosed in Table 2.

The obtained results have shown that the amount of RRT 0.95 at such conditions is about $1.73 \%$ (transformed in mass concentration, it is around $0.0173 \mathrm{mg} / \mathrm{mL}$ or $4.27 \cdot 10^{-5} \mathrm{~mol} /$ $\mathrm{dm}^{3}$ ). This sample was further analyzed with LC-MS for preliminary identification (Section 3.4).

3.2. Identification of Impurity RRT 0.95 in Bisoprolol FilmCoated Tablets by HPLC with Diode Array and Electrospray Mass Spectroscopy Detection. From the displayed UV spectra (Figure 3) of impurity with RRT 0.95 , it was seen that the spectrum obtained by HPLC/DAD/ESI-MS method had a different shape in relation to the spectral region of $225 \mathrm{~nm}$, due to fumaric acid presence in the mobile phase. The UV spectra obtained by HPLC/DAD/ESI-MS method for the target impurity retained the same characteristic absorption maximum on spectral region of $271 \mathrm{~nm}$. This was a good confirmation that it was the same impurity in both applied methods.
In order to study the molecular mass of Impurity RRT 0.95, as well as the structural preferences of this impurity, a dedicated HPLC study combined with diode array and electrospray ionization mass spectroscopy identification (HPLC/DAD/ESI-MS) was applied.

The obtained mass spectral data (MS and $\mathrm{MS}^{2}$ ) for bisoprolol and the target impurity with RRT 0.95 are given in Table 3 and the corresponding mass spectra are presented in Figure 4, respectively.

As can be seen from the obtained mass spectra (Table 3 and Figure 5), bisoprolol follows a distinct fragmentation pattern of the molecular ion (326 amu) to ions with $\mathrm{m} / z 222$ and 116 by a subsequent loss of 104 and 106 amu. The impurity with RRT 0.95 with molecular ion of $406 \mathrm{amu}$ follows a fragmentation pattern analogous to bisoprolol: fragments with $\mathrm{m} / z 302$ and 196 are obtained after the same losses of 104 and 106 amu as for bisoprolol (Figure 5).

The finding that the molecular mass of the target impurity $(m / z 406)$ and the fragments in $\mathrm{MS}^{2}(\mathrm{~m} / z 302$ and 196) have masses 80 amu higher compared to bisoprolol and its corresponding fragments implies that this impurity is a derivative of bisoprolol. Keeping in mind that this impurity increases in the binary mixture with calcium hydrogen phosphate, it is most probable that the linked group is actually a phosphate ion (with mass of $80 \mathrm{amu}$ ) attached to the 3-(isopropylamino) propan-2-ol part of the molecule of bisoprolol with a mass of 116 amu.

3.3. Structure Characterization of the Impurity with RRT 0.95. According to the previous experimental data obtained from the thermal degradation, another experimental setup was performed by introducing an equimolar amount of bisoprolol fumarate and calcium hydrogen phosphate. The binary mixture was subjected to stress conditions as presented in 
TABLE 2: Increase of the amount of RRT 0.95 before and after thermal degradation of drug product.

\begin{tabular}{lccc}
\hline & \multicolumn{3}{c}{ Finish drug product } \\
\hline Conditions of thermal degradation & Initial & $\left(80^{\circ} \mathrm{C}, 5 \mathrm{~h}\right)$ & $\left(80^{\circ} \mathrm{C}, 5 \mathrm{~d}\right)$ \\
$\%$ amount & $/$ & $0.50 \%$ & $1.73 \%$ \\
\hline
\end{tabular}

TABLE 3: UV absorbance and mass spectral data for bisoprolol and the target impurity.

\begin{tabular}{lccccc}
\hline Compounds & $t_{R} / \mathrm{min}$ & $\mathrm{UV}_{\max } / \mathrm{nm}$ & $\mathrm{MS}^{\mathrm{a}}$ ions $m / z$ & $\mathrm{MS}^{2}$ ions $m / z$ & $\mathrm{MS}^{3}$ ions $m / z$ \\
\hline Bisoprolol & 16.2 & 272 & $326^{\mathrm{b}}$ & $222 ; 116$ & 72 \\
Impurity RRT 0.95 & 15.9 & 272 & $406^{\mathrm{b}}$ & $302 ; 196$ & $284 ; 196$ \\
\hline
\end{tabular}

${ }^{\mathrm{a}}$ The most abundant ions are shown in bold. ${ }^{\mathrm{b}}$ Molecular ion obtained in positive ionization (protonation $\rightarrow \mathrm{M}+\mathrm{H}^{+}$).

Section 2.5.3 (thermal degradation study B). After the stress period, the amount of RRT 0.95 was found to be $2.5 \%$. The target impurity was isolated from the binary mixture by preparative HPLC and subsequently analyzed by means of 1D ${ }^{1} \mathrm{H}-\mathrm{NMR},{ }^{13} \mathrm{C}-\mathrm{NMR}$ spectroscopy, and ${ }^{31} \mathrm{P}-\mathrm{NMR}$ spectroscopy as well as two-dimensional HSQC ${ }^{1} \mathrm{H}_{-}{ }^{13} \mathrm{C}$ correlation. Spectra with respective data are included in Figures $6-10$. The structure of the impurity with RRT 0.95 was confirmed as phosphomonoester of bisoprolol (Figure 10), with relative molecular mass of 406 (positive ionization mode). The proposed mechanism of reaction includes esterification of bisoprolol with calcium hydrogen phosphate.

\subsection{Genotoxicity Prediction Using In Silico Methods}

3.4.1. Regulatory Framework. As per the regulatory guidelines $[3,20]$, the qualification of impurities is defined as the process of acquiring and evaluating data that establishes the biological safety of an individual degradation product or a given degradation profile at the level(s) specified and the first step in qualification of impurities is to conduct the minimum genotoxicity screening.

With the advances of computational toxicology and the strategies for nontesting approaches (e.g., REACH legislation), the use of in silico evaluations has rising potentials for qualification of impurities as well [21-23].

It is considered that the absence of structural alerts from two complementary (Q) SAR methodologies (expert rule-based and statistical) is sufficient to conclude that the impurity is of no mutagenic concern, and no further testing is recommended. Furthermore, an impurity with a structural alert that is shared (e.g., the same structural alert in the same position and chemical environment) with the drug substance or related compounds can be considered as nonmutagenic if the testing of such material in the bacterial mutagenicity assay was negative [20].

In order to qualify the impurity, the following in silico methodologies were applied: Toxtree (v. 2.6.13) and TEST (v. 4.1).

3.4.2. Toxtree Software. In Figures 11, 12, and 13, a comparative overview of the bisoprolol API and bisoprolol Impurity
RRT 0.95 is presented when evaluated with the three decision tree methods from Toxtree.

From the results presented in Figures 11 and 12, it can be concluded that, for the carcinogenicity and mutagenicity rule base as well as the rule base for Ames test, no structural alerts are identified either for bisoprolol API or for Impurity RRT 0.95. With regard to analysis using the decision tree for in vivo micronucleus assay in rodents, Figure 13, identical structural alerts are generated: $\mathrm{H}$-acceptor-path3-H-acceptor or structural alert 34 (SA_34), at the identical position in both structures. This alert is present both in bisoprolol API and in Impurity RRT 0.95 .

Genotoxicity studies performed on bisoprolol reveal that no evidence for genotoxic activity was observed with bisoprolol in vitro assays of gene mutation (reverse mutation in Salmonella Typhimurium, forward mutation in Chinese hamster V79 fibroblasts) or chromosomal damage (CHO cytogenetic assay). Negative findings were also obtained with bisoprolol in vivo assays of chromosomal damage (Chinese hamster bone marrow cytogenetic assay and the mouse micronucleus test) [24]. Therefore, the structural alert fired for the in vivo micronucleus assay in rodents is considered redundant since it is overridden by in vivo data.

3.4.3. TEST Software. Using the endpoint for Ames mutagenicity, according to the consensus method, the following results are generated for both bisoprolol API and the respective Impurity RRT 0.95: mutagenicity negative (Table 4).

From the results presented in Figure 14, it can be concluded that both bisoprolol and its related impurity with RRT 0.95 have no mutagenic potential when subjected to QSAR TEST software analysis that includes a statistical methodology for toxicity estimations.

3.4.4. QSAR Toolbox. Using the respective profilers, as presented in Table 5, the same results were obtained for both the parent compound and the respective impurity. Structural alert for in vivo mutagenicity (micronucleus) was fired for both compounds, which initially derive from the parent compound (Figure 14). By gathering data of category analogues from the relevant genotoxicity databases (bacterial mutagenicity ISSSTY, carcinogenicity/mutagenicity ISSCAN, 


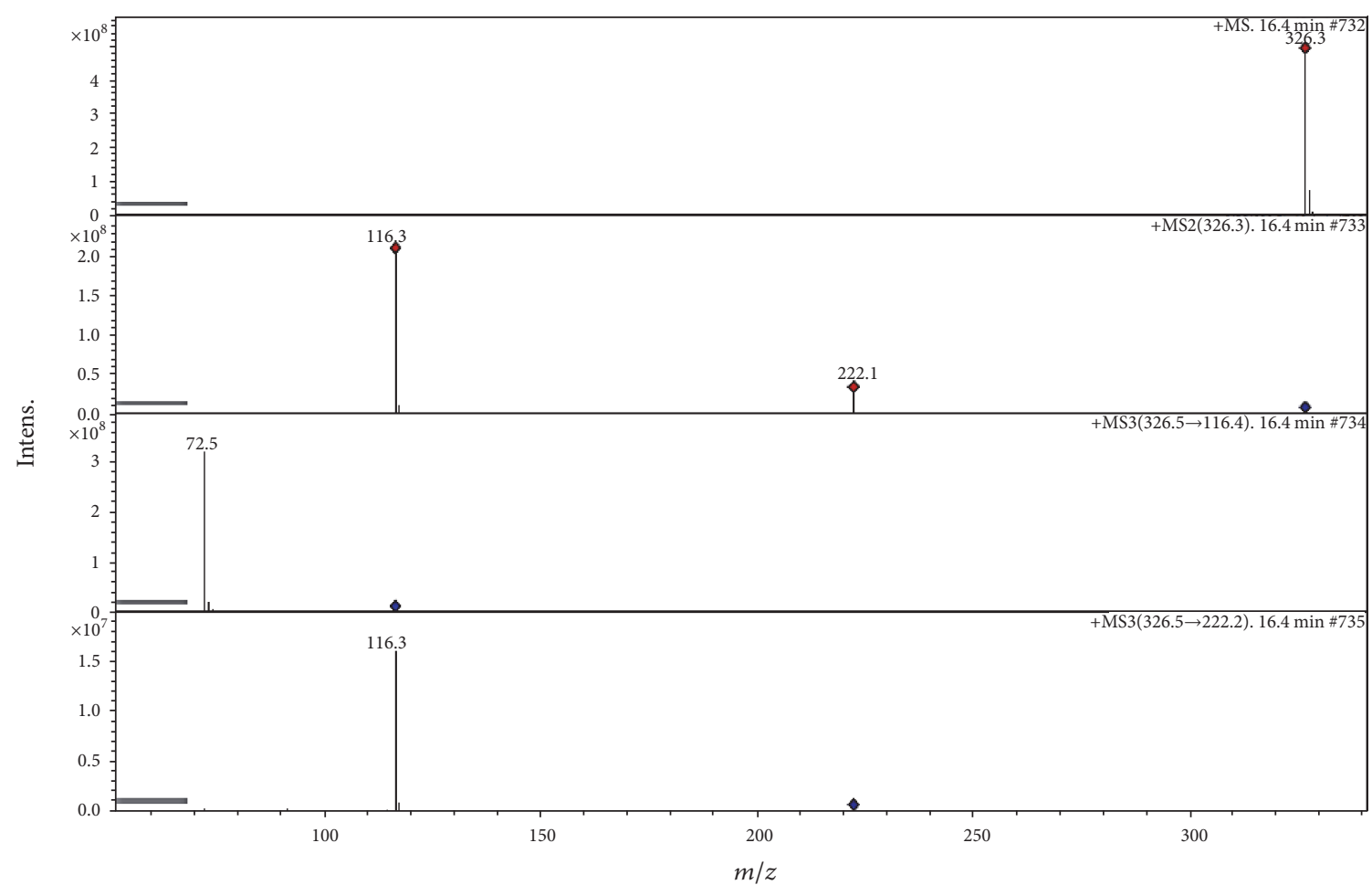

(a)

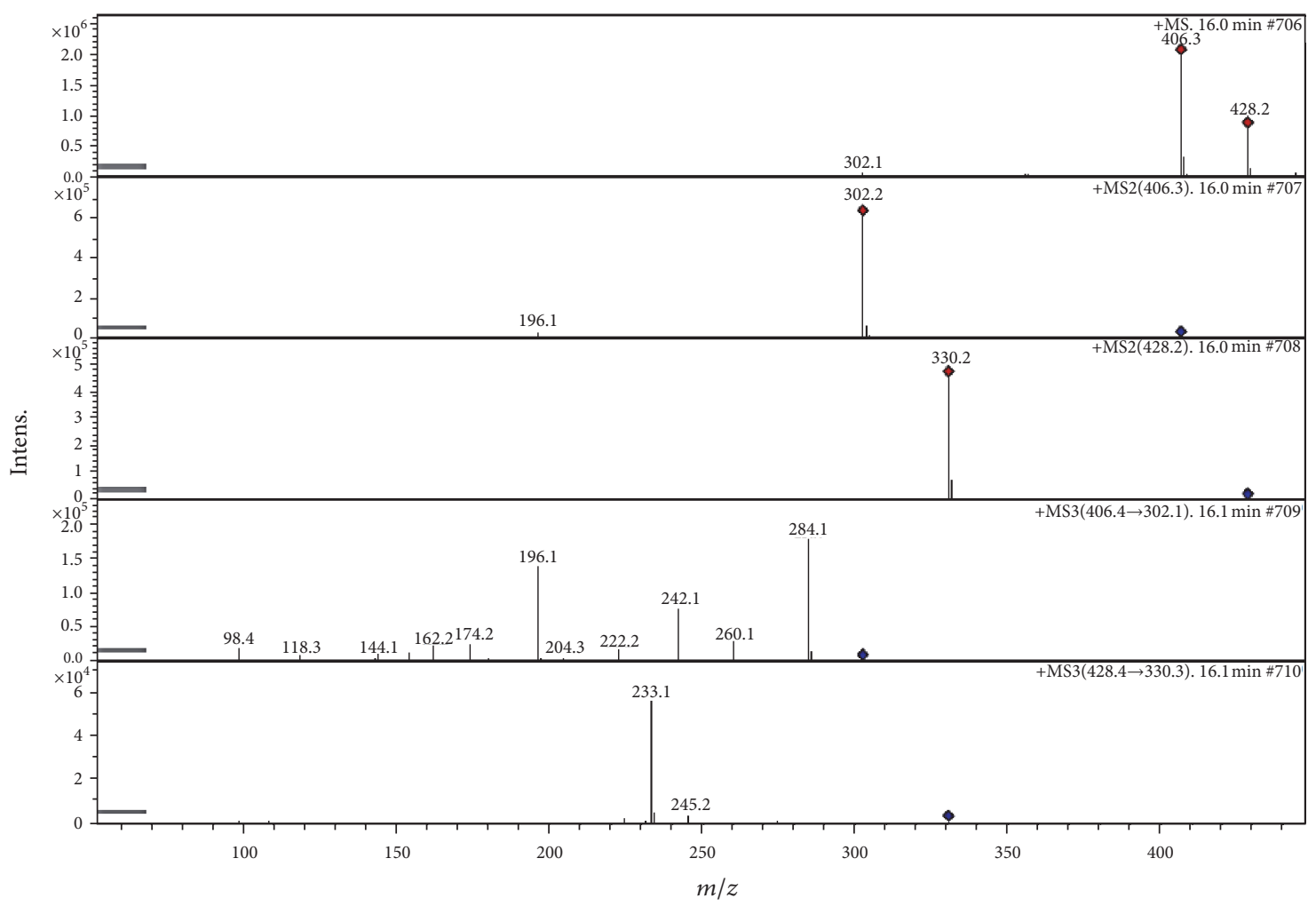

(b)

Figure 5: Mass spectra of (a) bisoprolol and (b) Imp. RRT 0.95. 
TABLE 4: Results from the TEST software using Ames mutagenicity endpoint with the consensus method.

\begin{tabular}{|c|c|c|c|c|c|}
\hline \multicolumn{3}{|c|}{ Prediction results } & \multicolumn{2}{|c|}{ Individual predictions } & \multirow[t]{2}{*}{ Test chemical } \\
\hline Endpoint & Experimental value & Predicted value & Method & Predicted value & \\
\hline \multicolumn{5}{|c|}{ (a) Bisoprolol API } & \\
\hline $\begin{array}{l}\text { Mutagenicity value } \\
\text { Mutagenicity result }\end{array}$ & $\begin{array}{l}\text { N/A } \\
\text { N/A }\end{array}$ & $\begin{array}{c}0.17 \\
\text { Mutagenicity negative }\end{array}$ & $\begin{array}{c}\text { Hierarchical clustering } \\
\text { FDA } \\
\text { Nearest neighbor }\end{array}$ & $\begin{array}{c}0.01 \\
N / A \\
0.33\end{array}$ & \\
\hline
\end{tabular}

(b) Imp. RRT 0.95

\begin{tabular}{|c|c|c|c|c|}
\hline $\begin{array}{l}\text { Mutagenicity value } \\
\text { Mutagenicity result }\end{array}$ & $\begin{array}{l}\text { N/A } \\
\text { N/A }\end{array}$ & $\begin{array}{c}0.32 \\
\text { Mutagenicity negative }\end{array}$ & $\begin{array}{c}\text { Hierarchical clustering } \\
\text { FDA } \\
\text { Nearest neighbor }\end{array}$ & $\begin{array}{l}0.42 \\
0.21 \\
0.33\end{array}$ \\
\hline
\end{tabular}

TABLE 5: QSAR Toolbox profiling of Bisoprolol API and Impurity RRT 0.95.

\begin{tabular}{lcc}
\hline Endpoint specific profilers & Bisoprolol API & Structural alerts \\
\hline Carcinogenicity (genotoxic and nongenotoxic) by ISS & No alert found & No alert found \\
DNA alerts for Ames by OASIS & No alert found & No alert found \\
DNA alerts for CA and MNT by OASIS & No alert found & No alert found \\
In vitro mutagenicity (Ames test) by ISS & No alert found & No alert found \\
In vivo chromosomal mutation & H-acceptor-path3-H-acceptor & H-acceptor-path3-H-acceptor \\
OncoLogic primary classification & No alert found & No alert found \\
\hline
\end{tabular}

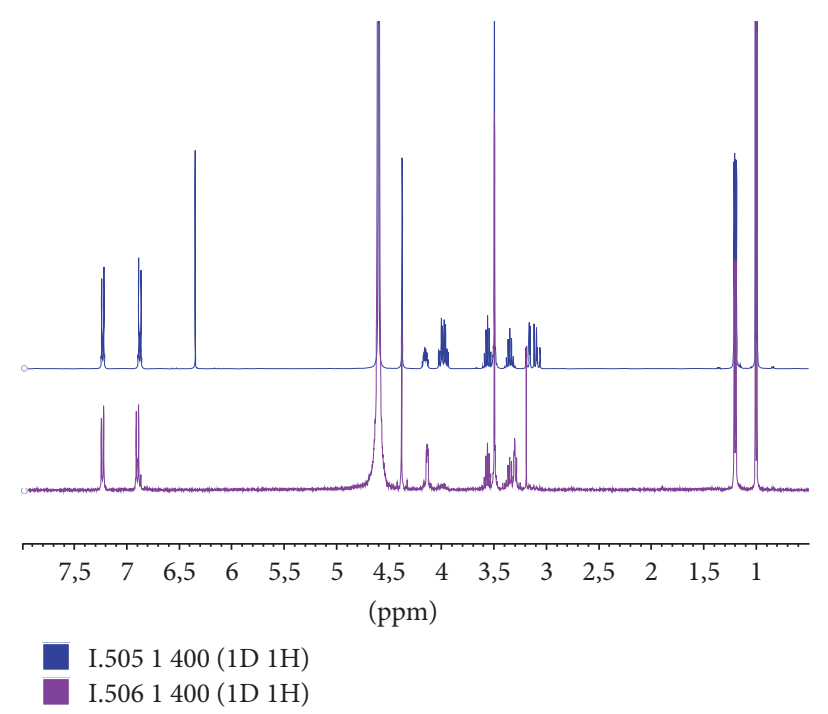

FIGURE 6: Superimposition of ${ }^{1} \mathrm{H}$ spectra of bisoprolol and Imp. RRT 0.95. 


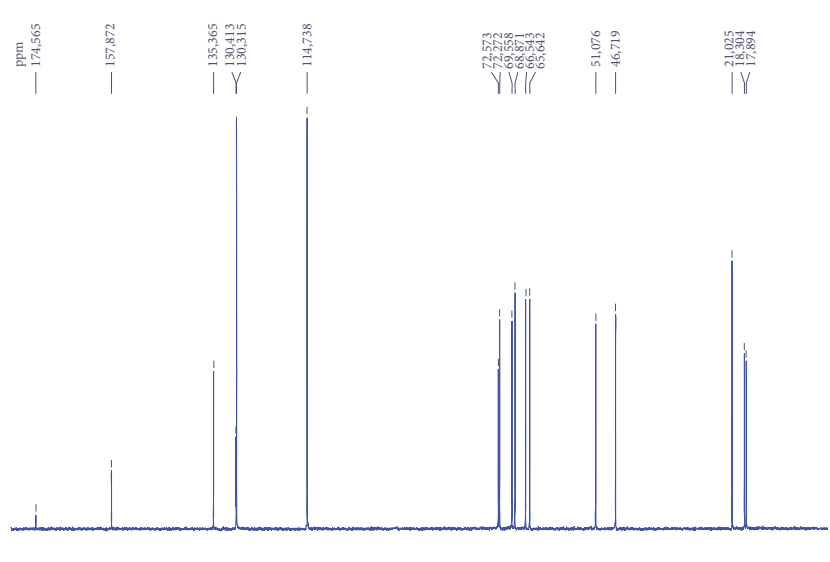

$17016015014013012011010090 \quad 80 \quad 70 \quad 60 \quad 50 \quad 40 \quad 30 \quad 20 \quad 10$ (ppm)

(a)

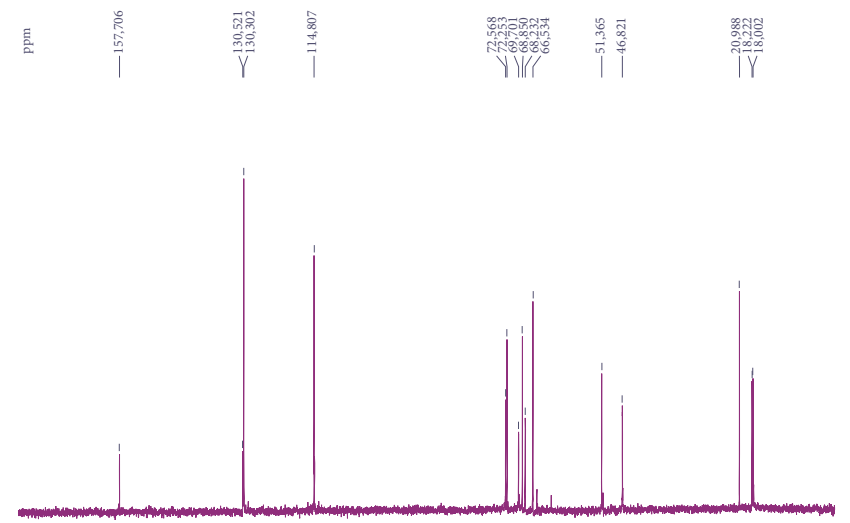

$170160150140130120110100 \quad 90 \quad 80 \quad 70 \quad 60 \quad 50 \quad 40 \quad 30 \quad 20 \quad 10$ (ppm)

(b)

Figure 7: $1 \mathrm{D}{ }^{13} \mathrm{C}$ spectrum of bisoprolol (a) and $1 \mathrm{D}{ }^{13} \mathrm{C}$ spectrum of Imp. RRT 0.95 (b).

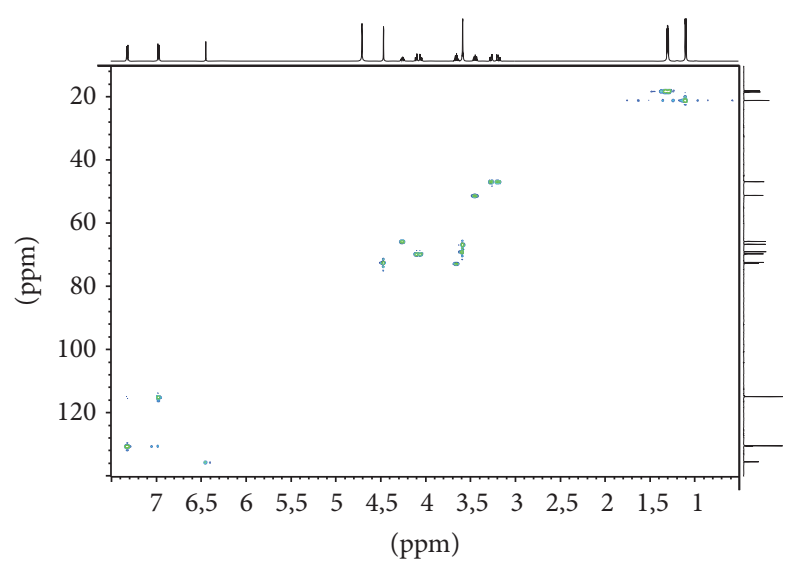

(a)

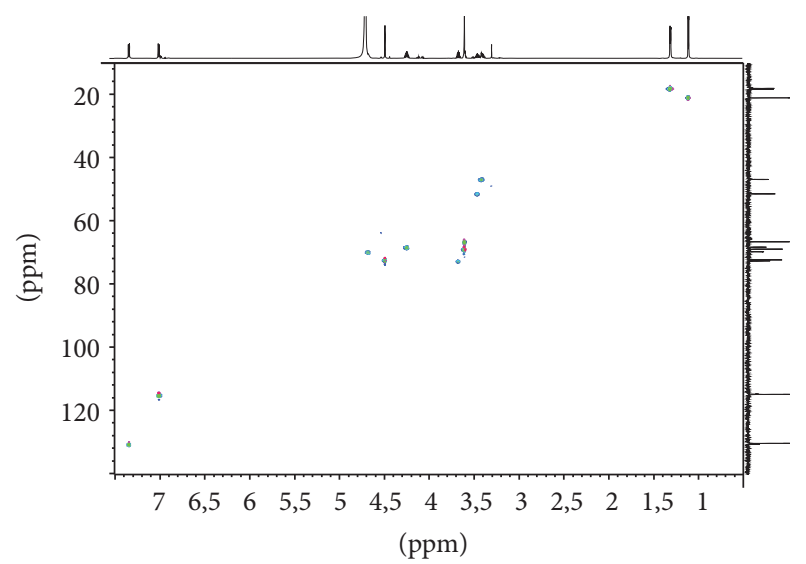

(b)

Figure 8: 2D ${ }^{13} \mathrm{C}$ spectrum of bisoprolol (a) and $2 \mathrm{D}{ }^{13} \mathrm{C}$ spectrum of Imp. RRT 0.95 (b).

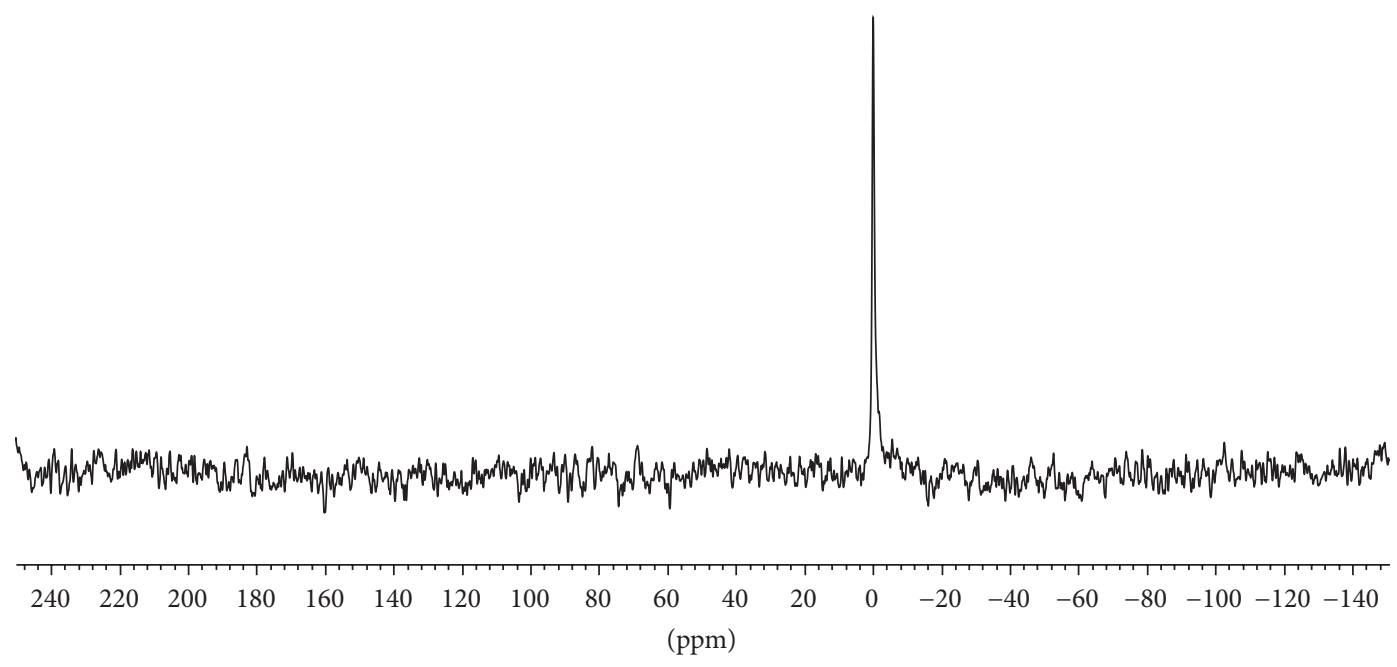

FIGURE 9: ${ }^{31} \mathrm{P}$ spectrum of Imp. RRT 0.95. 


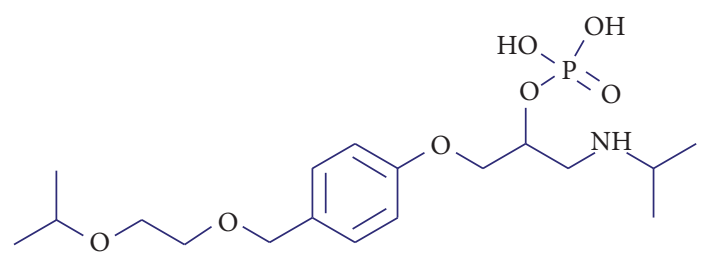

FIgURE 10: Phosphomonoester of bisoprolol (Imp. RRT 0.95).

genotoxicity OASIS, micronucleus ISSMIC, and micronucleus OASIS) [25], no experimental data was found for both the parent and the impurity. Despite not being part of the respective Toolbox databases, these studies have been performed for the parent compound and, as previously elaborated, are cited in the product monograph for bisoprolol [24]. Further analysis in Toolbox is not needed since existing experimental data for the parent compound override any in silico predictions in particular when the respective structural alert observed in a degradation product derives from the parent compound (as recommended in the ICH M7 guideline). As concluded for the similar analysis in Toxtree, the structural alert fired for the in vivo mutagenicity is considered redundant since it is overridden by negative in vivo data.

\section{Conclusions}

An unidentified impurity with RRT 0.95 was structurally identified and characterized by mass spectroscopy, ${ }^{1} \mathrm{H}-\mathrm{NMR}$ and ${ }^{13} \mathrm{C}$-NMR spectroscopy, and ${ }^{31} \mathrm{P}$ spectroscopy.

Force degradation study was established based on the knowledge of the drug substance behavior and the internal stability of the molecule of bisoprolol fumarate. Thermal degradation of the finished product, binary mixtures of active ingredient, and each excipient included in the formulation was performed. The result of the analysis showed the increase of the proportion for the unidentified impurity with RRT 0.95 with temperature, in combination with calcium hydrogen phosphate.

From the obtained data for the unidentified impurity, the samples of binary mixture of bisoprolol fumarate and calcium hydrogen phosphate anhydrous were used for further research and designing towards structural characterization of the unidentified impurity with RRT 0.95 .

Impurity RRT 0.95 was isolated from the binary mixture and subsequently analyzed by $1 \mathrm{D}{ }^{1} \mathrm{H}-\mathrm{NMR}$ and ${ }^{13} \mathrm{C}-\mathrm{NMR}$ spectroscopy, 2D HSQC ${ }^{1} \mathrm{H}_{-}{ }^{13} \mathrm{C}$ spectroscopy, mass spectroscopy, and ${ }^{31} \mathrm{P}$ spectroscopy. The structure of Impurity RRT 0.95 was elucidated as phosphomonoester of bisoprolol with relative molecular mass of 406 (positive ionization mode).

Since bisoprolol fumarate is a cardiovascular (beta blocking) agent intended for long-term treatment that includes regular daily intake as a tablet dosage form, it is crucial to have stringent control of the quality of the product particularly in terms of controlling the levels of impurities and degradation products. In that sense, qualification of an unknown impurity/degradation product as a means of indicating biological safety is a quality prerequisite for providing a product that is efficient and safe. Therefore, in order to properly qualify an unidentified impurity, the first step was full structural characterization followed by toxicological evaluation. From the evaluations performed, it can be concluded that the structurally identified impurity with RRT 0.95 has no mutagenic or carcinogenic potential.

The study encompasses in-depth research for full characterization of an unidentified impurity with a better understanding on the degradation reaction mechanism that leaves further possibilities for continuous improvement of the quality of the product.

\section{Abbreviations \\ RRT: $\quad$ Relative retention time \\ HPLC: High performance liquid chromatography \\ DAD: Diode array detector \\ ESI-MS: Electrospray ionization mass spectrometry \\ NMR: Nuclear magnetic resonance \\ API: Active pharmaceutical ingredient \\ ICH: International Council for Harmonisation.}

\section{Conflicts of Interest}

The authors declare that there are no conflicts of interest regarding the publication of this paper.

\section{Authors' Contributions}

The manuscript was written through contributions of all authors. All authors have given approval to the final version of the manuscript.

\section{Acknowledgments}

This research has been supported by Alkaloid AD Skopje, Macedonia. The authors would like to thank their colleagues Biljana Sapkareva, (Eng.Tech.), Ana Georgieva, (B.Pharm.), and Professor Dr. Marina Stefova for the professional support and scientific input. Professor Dr. Aneta Dimitrovska is acknowledged for critical reading of the manuscript. The authors would also like to thank NMRTEC, France, for providing the NMR spectral analyses. 


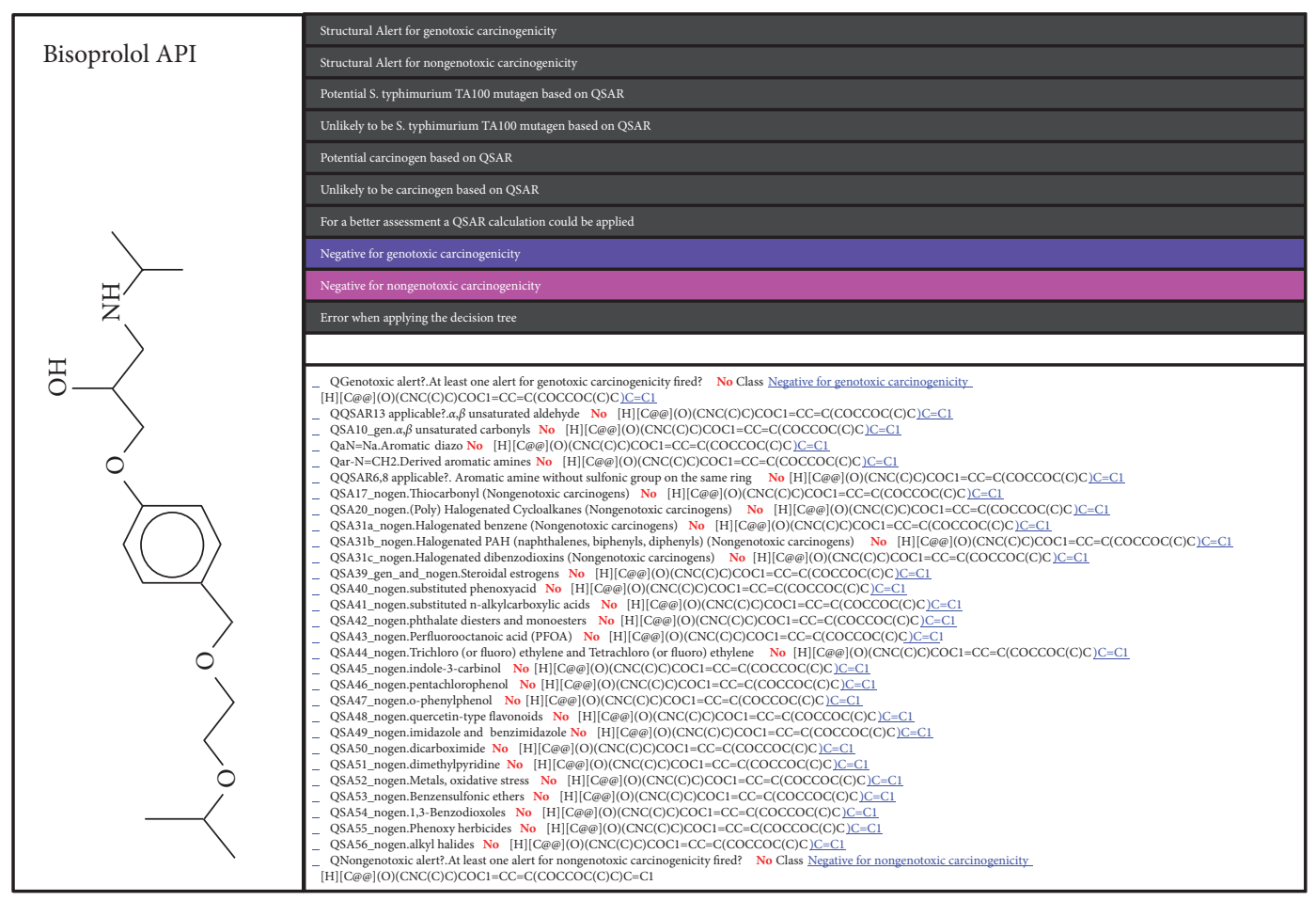

(a)

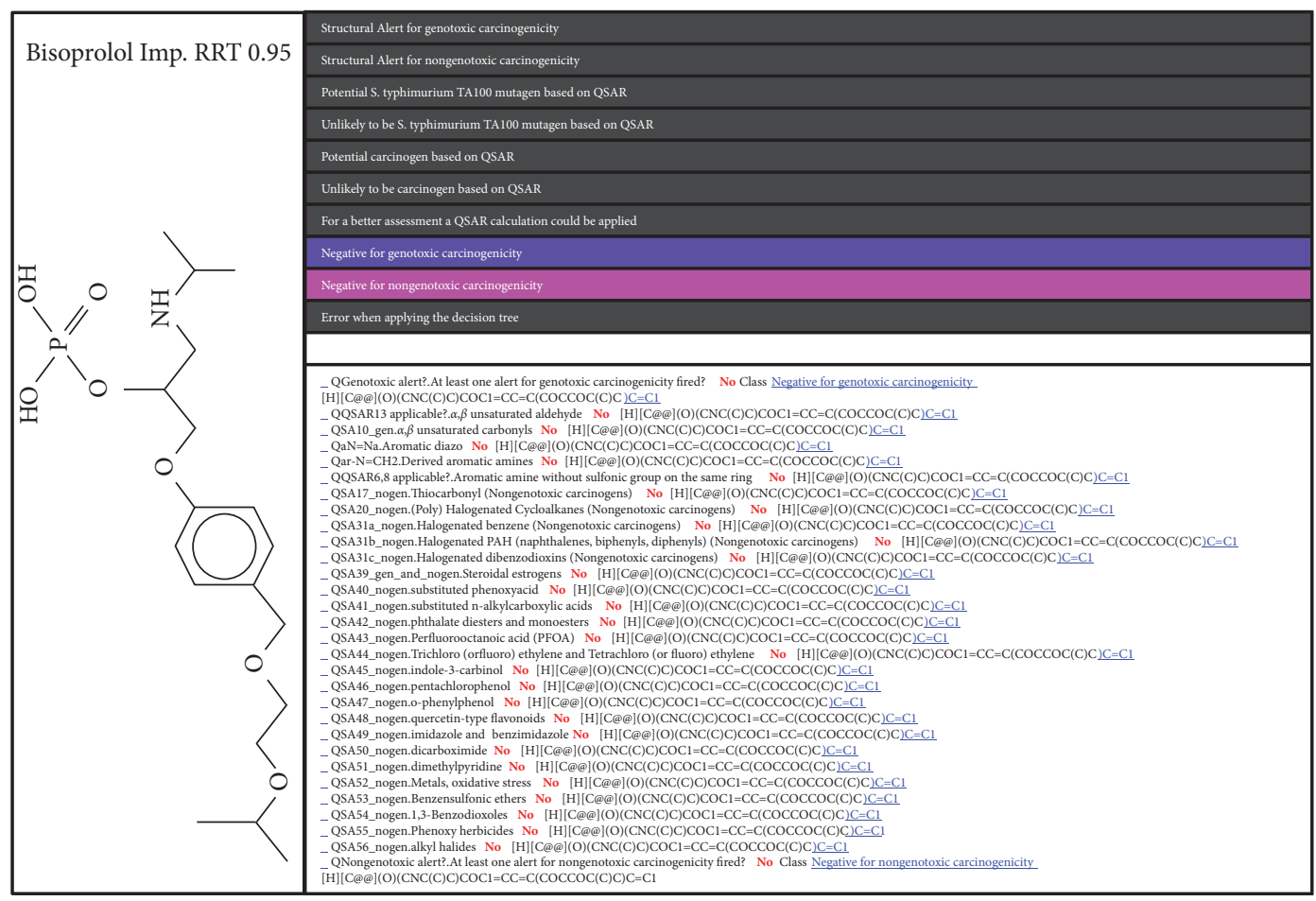

(b)

FIGURE 11: Results from the decision tree for estimating carcinogenicity (genotoxic and nongenotoxic) and mutagenicity: bisoprolol API (a) and bisoprolol Imp. RRT 0.95 (b). 


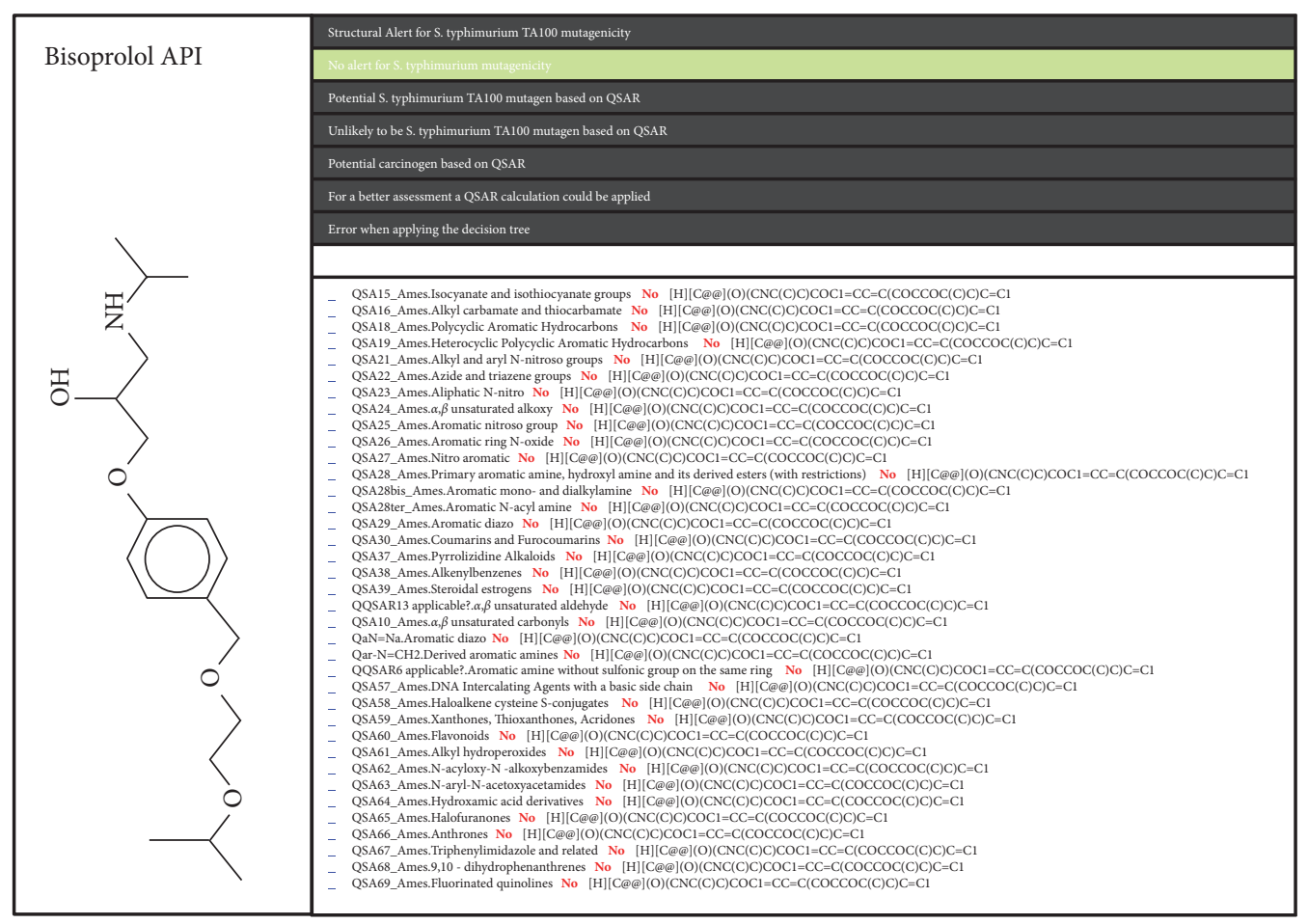

(a)

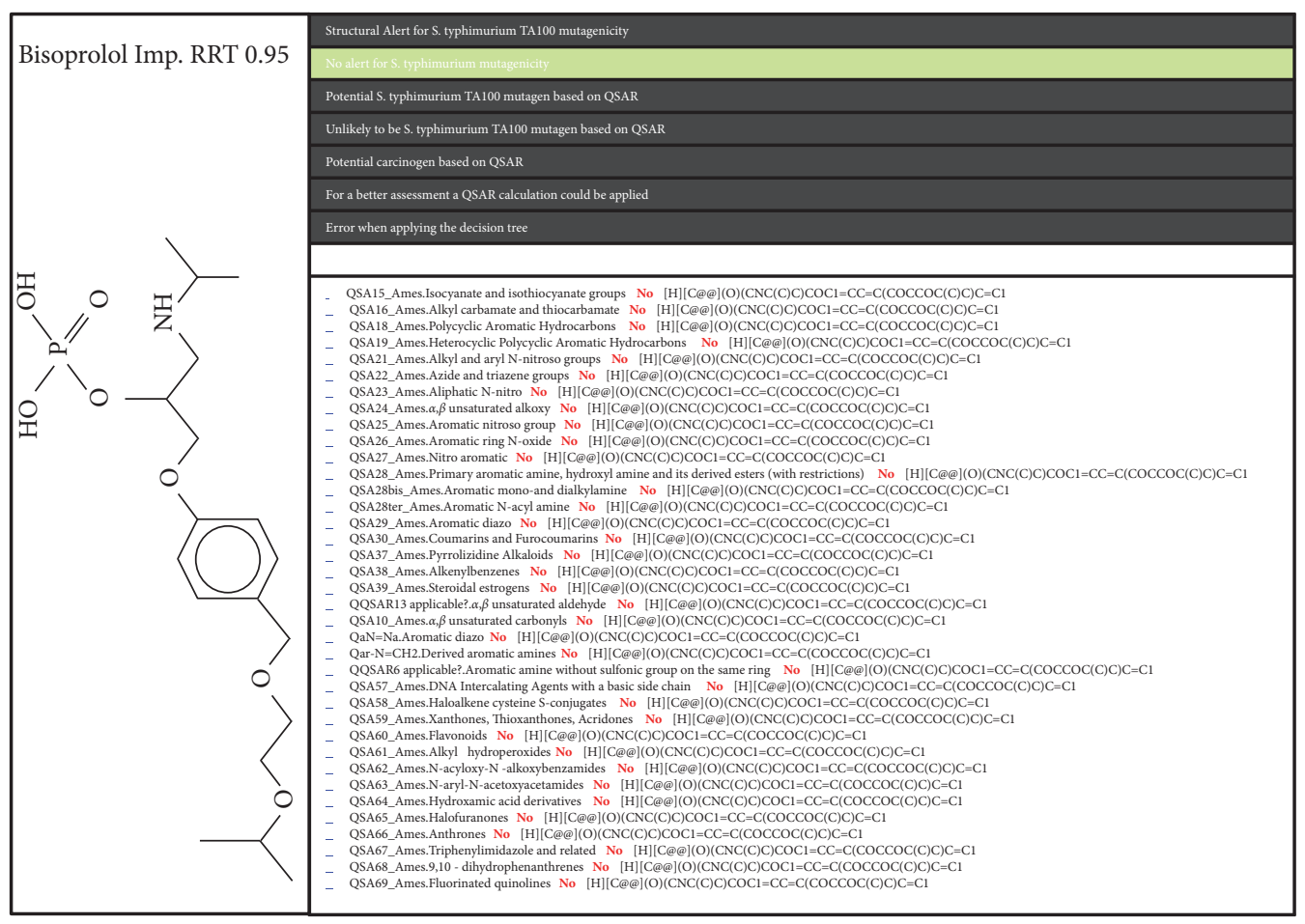

(b)

FIGURE 12: Results from the decision tree for estimating in vitro mutagenicity (Ames test): bisoprolol API (a) and bisoprolol Imp. RRT 0.95 (b). 


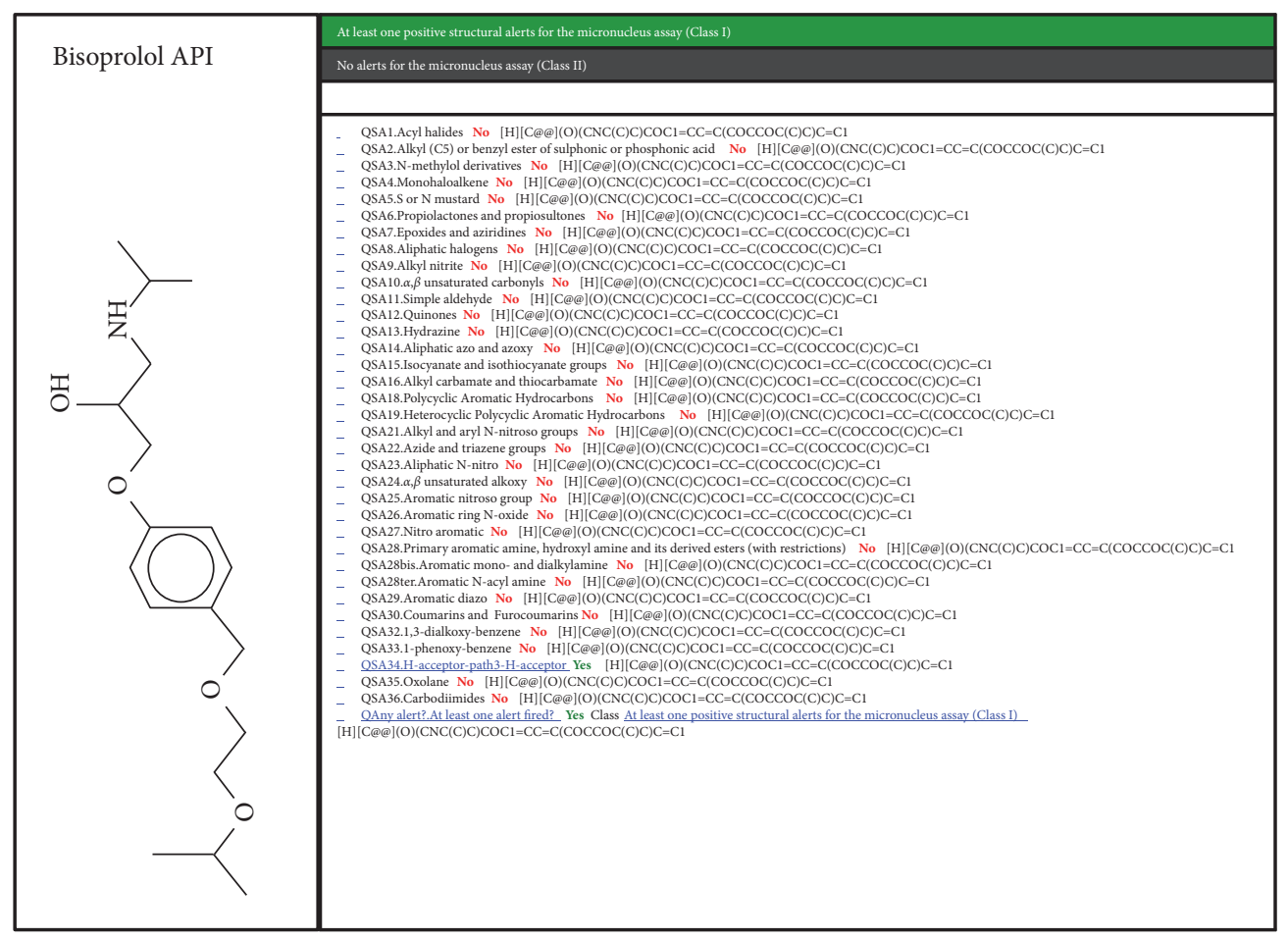

(a)

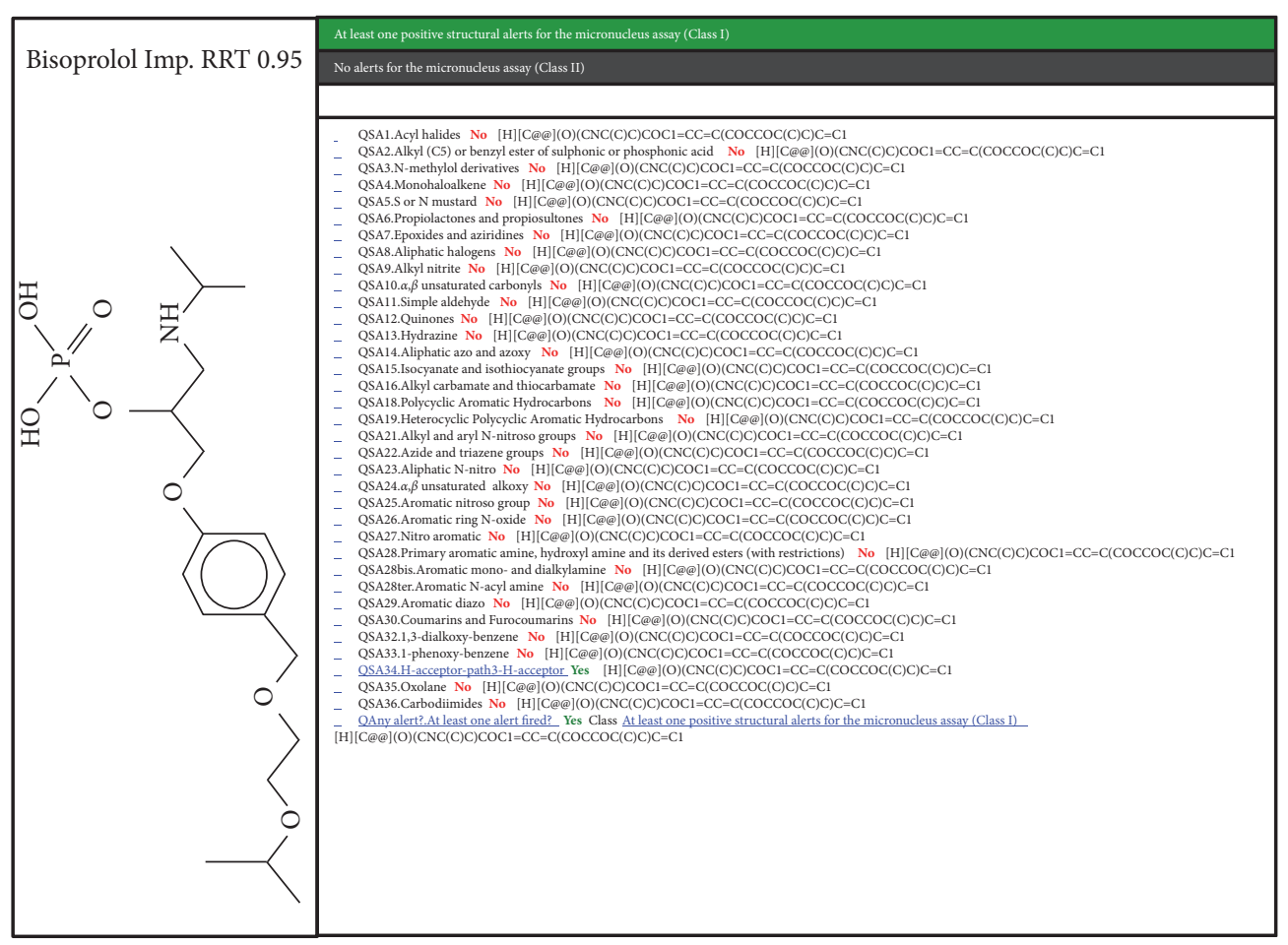

(b)

FIGURE 13: Results from the decision tree for estimating structure alerts for the in vivo micronucleus assay in rodents: bisoprolol API (a) and bisoprolol Imp. RRT 0.95 (b). 


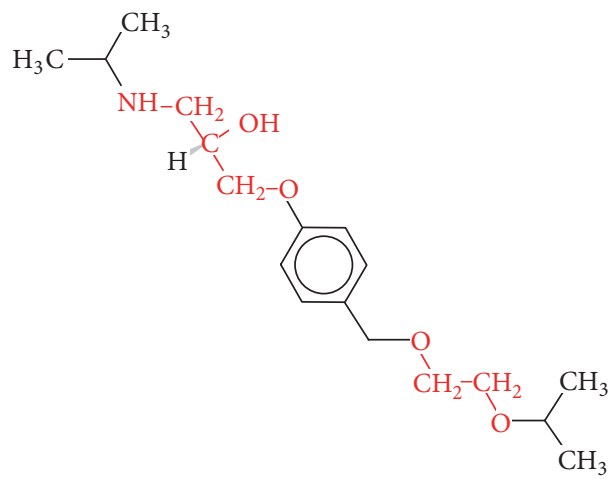

(a)

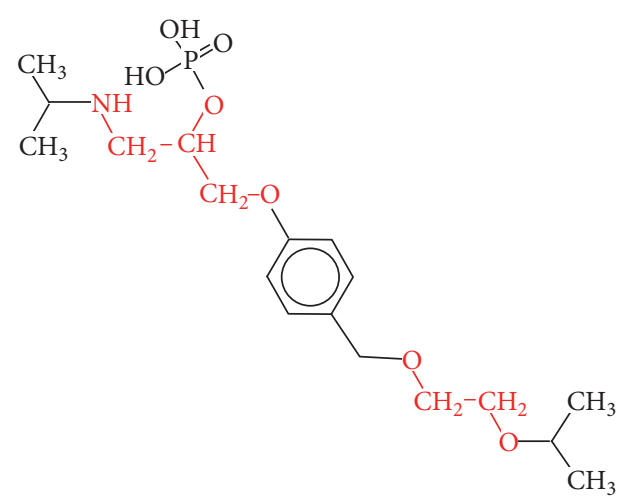

(b)

FIGURE 14: Structural alerts for in vivo mutagenicity (micronucleus assay): $\mathrm{H}$-acceptor-path3-H-acceptor. (a) Bisoprolol API and (b) Impurity RRT 0.95 .

\section{References}

[1] S. Görög, Identification and Determination of Impurities in Drugs, Elsevier Science B.V. Publisher, Amsterdam, The Netherlands, 2000.

[2] K. Ahuja and K. M. Alsante, Handbook of Isolation and Characterization of Impurities in Pharmaceuticals, Elsevier Science, Atlanta, Ga, USA, 2003.

[3] $\mathrm{ICH}$, "Assessment and control of DNA reactive (mutagenic) impurities in pharmaceuticals to limit potential carcinogenic risk, Current Step 2," in Proceedings of the International Conference on Harmonization (ICH) of Technical Requirements for Registration of Pharmaceuticals for Human Use, Topic M7, 2013.

[4] N. A. Charoo, A. A. A. Shamsher, L. Y. Lian et al., "Biowaiver monograph for immediate-release solid oral dosage forms: bisoprolol fumarate," Journal of Pharmaceutical Sciences, vol. 103, no. 2, pp. 378-391, 2014.

[5] European Pharmacopoeia 6th Edition Supplement 6.1 (04/ 2008:1710), Monograph for Bisorprolol Fumarate, Europe 2008, 2008.

[6] J. Ermer, "The use of hyphenated LC-MS technique for characterisation of impurity profiles during drug development," Journal of Pharmaceutical and Biomedical Analysis, vol. 18, no. 4-5, pp. 707-714, 1998.

[7] E. C. Nicolas and T. H. Scholz, "Active drug substance impurity profiling: part II. LC/MS/MS fingerprinting," Journal of Pharmaceutical and Biomedical Analysis, vol. 16, no. 5, pp. 825-836, 1998.

[8] W. M. A. Niessen, "State-of-the-art in liquid chromatographymass spectrometry," Journal of Chromatography A, vol. 856, no. 1-2, pp. 179-197, 1999.

[9] W. F. Smyth, "Recent studies on the electrospray ionisation mass spectrometric behaviour of selected nitrogen-containing drug molecules and its application to drug analysis using liquid chromatography-electrospray ionisation mass spectrometry," Journal of Chromatography B, vol. 824, no. 1-2, pp. 1-20, 2005.

[10] W. F. Smyth, C. Joyce, V. N. Ramachandran, E. O'Kane, and D. Coulter, "Characterisation of selected hypnotic drugs and their metabolites using electrospray ionisation with ion trap mass spectrometry and with quadrupole time-of-flight mass spectrometry and their determination by liquid chromatographyelectrospray ionisation-ion trap mass spectrometry," Analytica Chimica Acta, vol. 506, no. 2, pp. 203-214, 2004.
[11] International Conference on Harmonization (ICH) of Technical Requirements for registration of Pharmaceuticals for Human Use, Topic Q1A(R2), Stability Testing of New Drug Substances and Products, 2003.

[12] R. Benigni, C. Bossa, N. Jeliazkova, T. Netzeva, and A. Worth, "The Benigni/Bossa rulebase for mutagenicity and carcinogenicity\&a module of Toxtree," European Commission Report, 2013.

[13] R. Benigni, C. Bossa, and O. Tcheremenskaia, "In vitro cell transformation assays for an integrated, alternative assessment of carcinogenicity: a data-based analysis," Mutagenesis, vol. 28, no. 1, pp. 107-116, 2013.

[14] R. Benigni, C. Bossa, O. Tcheremenskaia, and A. Worth, "Development of structural alerts for the in vivo micronucleus assay in rodents," European Commission Report, 2009.

[15] US Environmental Protection Agency, Toxicity Estimation Software Tool (TEST), QSAR Toolbox 3.3, 2015.

[16] T. M. Martin, P. Harten, R. Venkatapathy, S. Das, and D. M. Young, "A hierarchical clustering methodology for the estimation of toxicity", Toxicology Mechanisms and Methods, vol. 18, no. 2-3, pp. 251-266, 2008.

[17] User's Guide for T.E.S.T., A Program to Estimate Toxicity from Molecular Structure, U.S. Environmental Protection Agency, 2012.

[18] N. George, "Forced degradation as an integral part of HPLC stability-indicating method development," Drug Delivery Technology, vol. 10, no. 5, 2010.

[19] K. Huynh-Ba, Handbook of Stability Testing in Pharmaceutical Development, Springer Science \& Business Media, New York, NY, USA, 2009.

[20] International Conference on Harmonization (ICH) of Technical Requirements for registration of Pharmaceuticals for Human Use, Topic Q3B(R2), Impurities in New Drug Products, 2006.

[21] A. Sutter, A. Amberg, S. Boyer et al., "Use of in silico systems and expert knowledge for structure-based assessment of potentially mutagenic impurities," Regulatory Toxicology and Pharmacology, vol. 67, no. 1, pp. 39-52, 2013.

[22] J. F. Contrera, "Validation of Toxtree and SciQSAR in silico predictive software using a publicly available benchmark mutagenicity database and their applicability for the qualification of impurities in pharmaceuticals," Regulatory Toxicology and Pharmacology, vol. 67, no. 2, pp. 285-293, 2013. 
[23] R. Benigni and C. Bossa, "Mechanisms of chemical carcinogenicity and mutagenicity: a review with implications for predictive toxicology," Chemical Reviews, vol. 111, no. 4, pp. 2507-2536, 2011.

[24] Product Monograph, APO-Bisoprolol Tablets, Apotex Inc, Macquarie Park, Australia, 2014.

[25] QSAR Toolbox Version 3.3, http://www.qsartoolbox.org. 

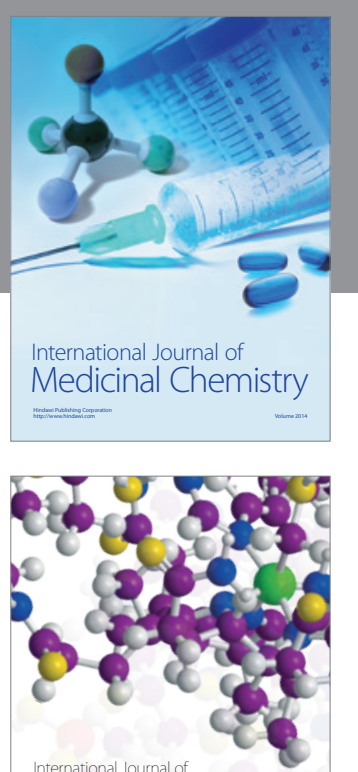

Carbohydrate Chemistry

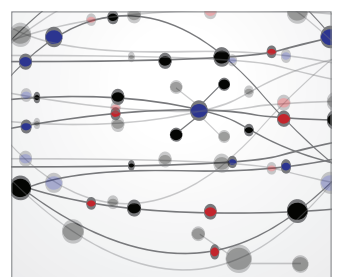

The Scientific World Journal
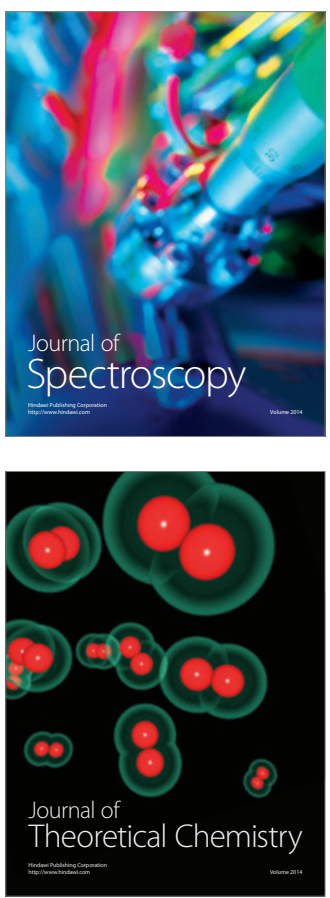
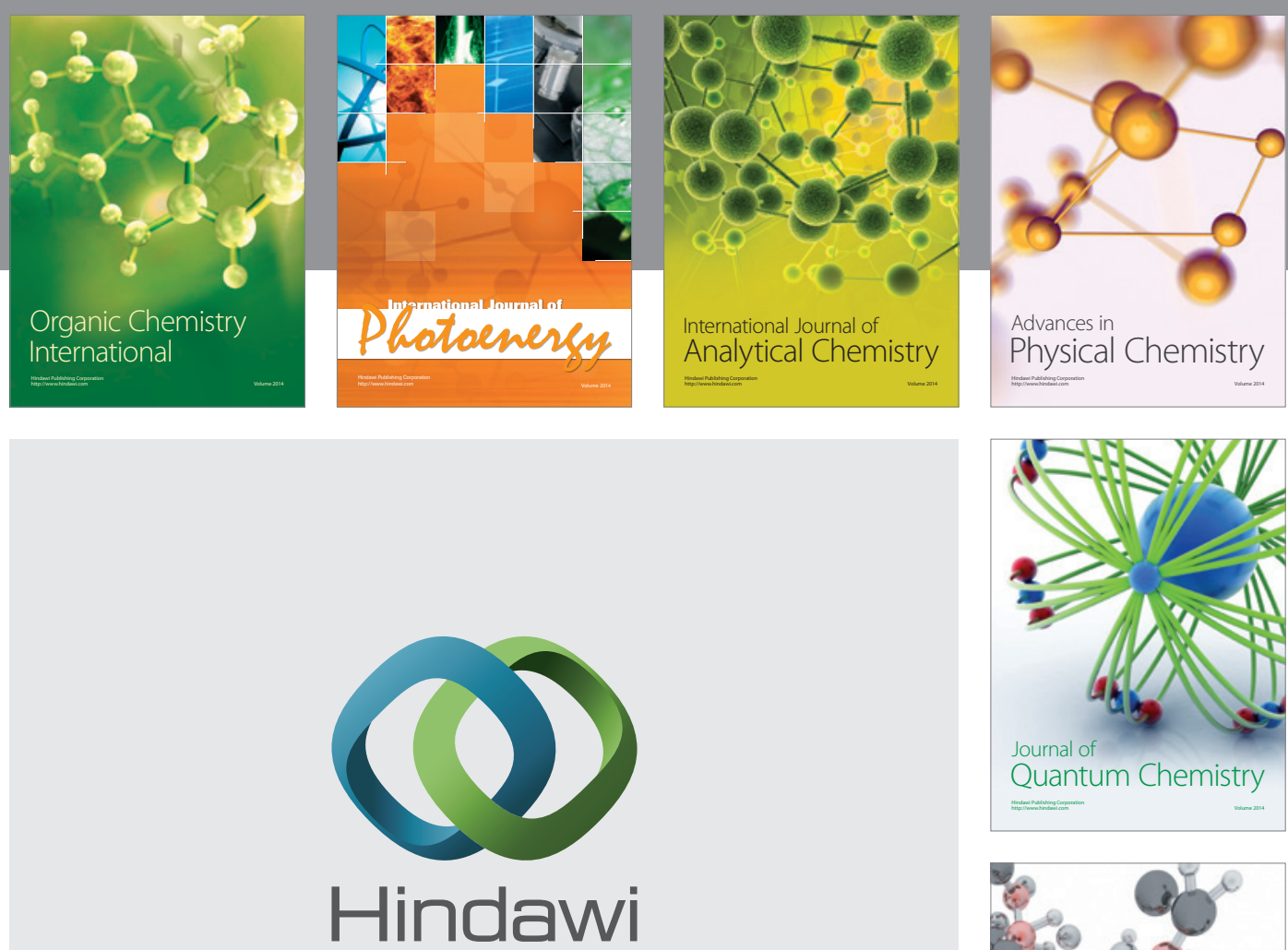

Submit your manuscripts at

https://www.hindawi.com

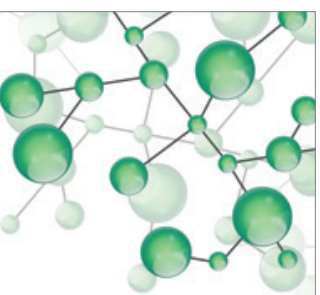

International Journal of

Inorganic Chemistry
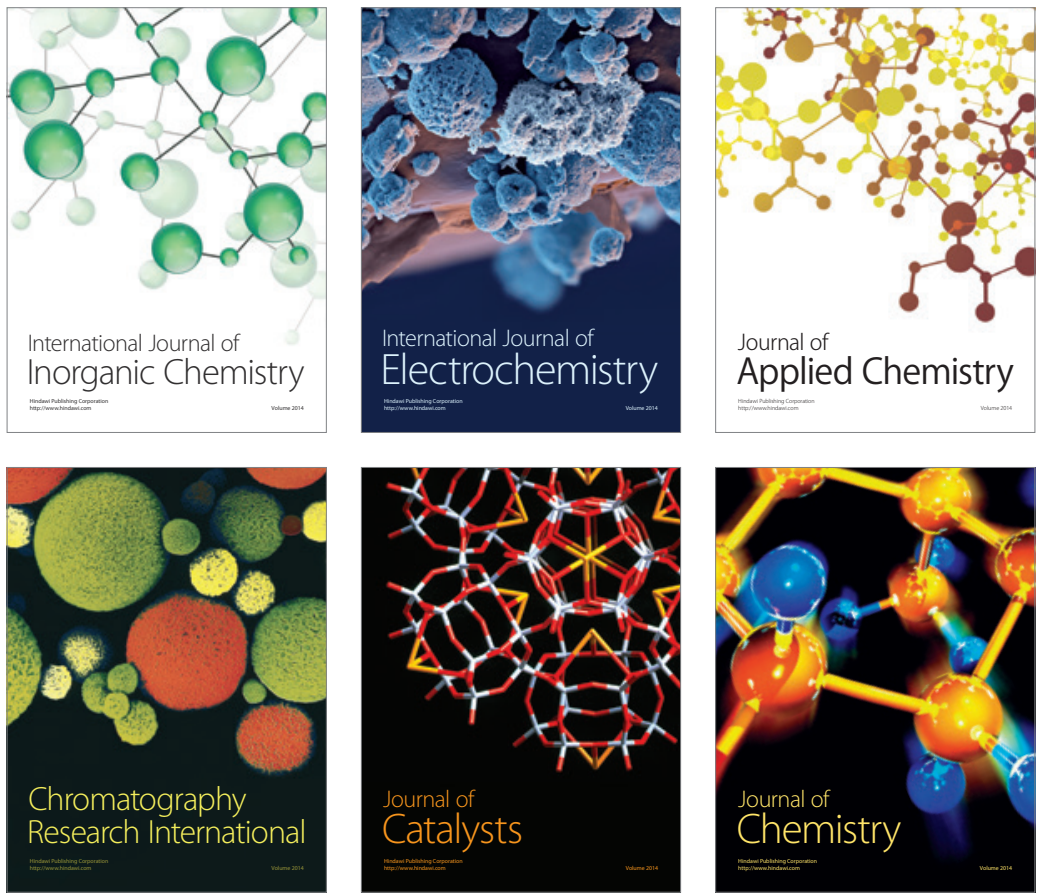

Journal of

Applied Chemistry
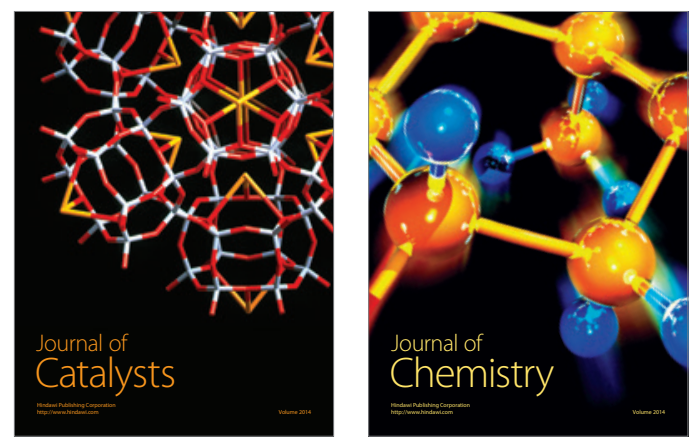
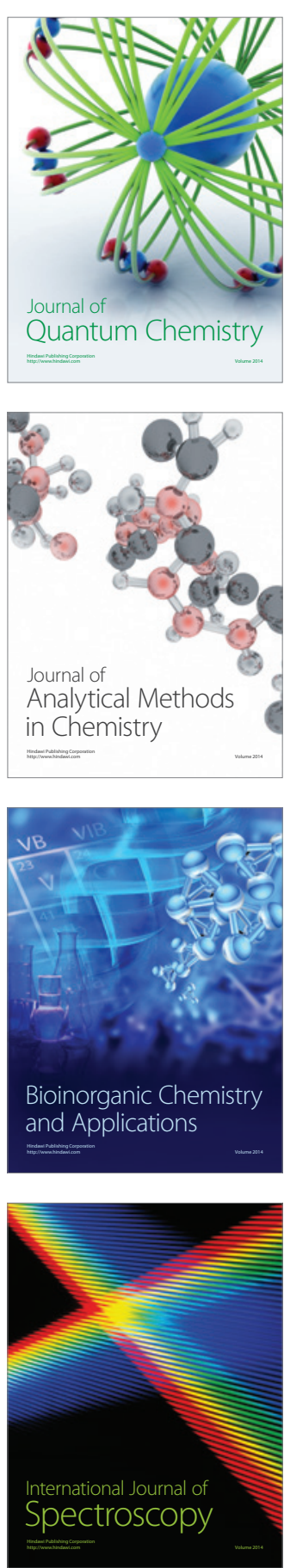